\title{
Glial Cell Line-derived Neurotrophic Factor-overexpressing Human Neural Stem/Progenitor Cells Enhance Therapeutic Efficiency in Rat with Traumatic Spinal Cord Injury
}

\author{
Kyujin Hwang ${ }^{1,2 \dagger}$, Kwangsoo Jung ${ }^{3 \dagger}$, Il-Sun Kim², Miri Kim², Jungho Han ${ }^{3}$, \\ Joohee Lim ${ }^{3}$, Jeong Eun Shin ${ }^{3}$, Jae-Hyung Jang ${ }^{4}$ and Kook In Park ${ }^{1,2,3 *}$ \\ ${ }^{1}$ Brain Korea 21 Plus Project for Medical Science, Yonsei University College of Medicine, \\ ${ }^{2}$ Yonsei Biomedical Research Institute, Yonsei University College of Medicine, ${ }^{3}$ Department of Pediatrics, \\ Severance Children's Hospital, Yonsei University College of Medicine, Seoul 03722, Korea, \\ ${ }^{4}$ Department of Chemical and Biomolecular Engineering, Yonsei University, Seoul 03722, Korea
}

\begin{abstract}
Spinal cord injury (SCI) causes axonal damage and demyelination, neural cell death, and comprehensive tissue loss, resulting in devastating neurological dysfunction. Neural stem/progenitor cell (NSPCs) transplantation provides therapeutic benefits for neural repair in SCI, and glial cell linederived neurotrophic factor (GDNF) has been uncovered to have capability of stimulating axonal regeneration and remyelination after SCI. In this study, to evaluate whether GDNF would augment therapeutic effects of NSPCs for SCI, GDNF-encoding or mock adenoviral vector-transduced human NSPCs (GDNF-or Mock-hNSPCs) were transplanted into the injured thoracic spinal cords of rats at 7 days after SCI. Grafted GDNFhNSPCs showed robust engraftment, long-term survival, an extensive distribution, and increased differentiation into neurons and oligodendroglial cells. Compared with Mock-hNSPC- and vehicle-injected groups, transplantation of GDNF-hNSPCs significantly reduced lesion volume and glial scar formation, promoted neurite outgrowth, axonal regeneration and myelination, increased Schwann cell migration that contributed to the myelin repair, and improved locomotor recovery. In addition, tract tracing demonstrated that transplantation of GDNF-hNSPCs reduced significantly axonal dieback of the dorsal corticospinal tract (dCST), and increased the levels of dCST collaterals, propriospinal neurons (PSNs), and contacts between dCST collaterals and PSNs in the cervical enlargement over that of the controls. Finally grafted GDNF-hNSPCs substantially reversed the increased expression of voltage-gated sodium channels and neuropeptide $\mathrm{Y}$, and elevated expression of GABA in the injured spinal cord, which are involved in the attenuation of neuropathic pain after SCI. These findings suggest that implantation of GDNF-hNSPCs enhances therapeutic efficiency of hNSPCs-based cell therapy for SCI.
\end{abstract}

Key words: Spinal cord injuries, Glial cell line-derived neurotrophic factor, Neural stem/progenitor cells, Cell-based therapy, Mechanical allodynia, Paraplegia

Received September 18,2019, Revised December 4,2019,

Accepted December 6, 2019

* To whom correspondence should be addressed.

TEL: 82-10-9091-9231, FAX: 82-2-393-9118

e-mail:kipark@yuhs.ac

These authors contributed equally.

\section{INTRODUCTION}

Spinal cord injury (SCI) is a devastating neurological disorder that leads to motor, sensory, or autonomic dysfunction, and is associated with high rates of mortality and complications including bladder infection, renal failure, cardiovascular disease, and respiratory dysfunctions. There are approximately 180,000 new cases of SCI worldwide per year caused by traffic accidents, sports injuries, and other traumatic events according to an epidemiologic study 
[1]. Traumatic SCI causes extensive spinal tissue damage, loss of neural cells, axonal injury, demyelination, and glial scar formation, which adversely impact overall patient outcomes and place financial burdens on healthcare systems. However, there is no standard and efficient management approach for SCI patients [2,3].

Neural stem/progenitor cells (NSPCs) transplanted into the damaged spinal cord robustly engraft, migrate toward the lesion site, differentiate into all three types of neural lineage cells, integrate into host tissues, provide trophic/anti-inflammatory factors, regenerate lost neural circuits, and remyelinate injured axons, all of which are functionally beneficial following SCI [4-12]. In addition, human fetal brain or spinal cord-derived NSPCs (hNSPCs) implanted into animal models of SCI incorporate into the host neural circuitry and improve locomotor function [13-17]. Recent clinical trials demonstrate that transplantation of hNSPCs is safe and potentially efficacious for treatment of SCI [18-20]. However, implanted NSPCs exhibit poor survival, integration, differentiation, and functional neural connections in the inhospitable environment of the injured central nervous system (CNS) [4, 5, 21, 22]. Thus, the therapeutic efficiency of NSPCs for SCI is insufficient.

Glial cell line-derived neurotrophic factor (GDNF) has developmental, neuroprotective and neurotrophic effects on some types of neurons in the CNS and peripheral nervous system [23, 24]. Administration of GDNF reduces motor neuron death, secondary damage, and white matter atrophy in the spinal cord, increases neuronal survival and the number of spared neuronal fibers, promotes the survival and proliferation of Schwann cells (SCs), and improves motor function post-SCI [25-29]. The methods by which GDNF is delivered to the injured spinal cord vary widely, and include osmotic minipumps, direct injection, GDNF-releasing guidance channels, guidance channels containing SCs and GDNF, injectable GDNF-embedded hydrogels, GDNF-releasing biodegradable polymeric microspheres, hydrogel scaffolds containing GDNF and SCs, and genetically modified GDNF-secreting cells $[29,30]$. Recent studies suggest that combinational treatments using many types of genetically modified cells, including SCs, olfactory ensheathing cells, immortalized mouse neural stem cells, fibroblasts, bone marrow-derived mesenchymal stem cells (MSCs), and umbilical cord blood mononuclear cells or MSCs, have varying therapeutic potentials for SCI $[29,30]$.

We previously reported that human fetal brain-derived primary NSPCs implanted into the injured rat spinal cord robustly engraft, migrate, integrate with host cells, and differentiate into neuronal and glial cells, although most remain immature cells, resulting in improved locomotor recovery. However, a clinical trial in which hNSPC were transplanted into patients with traumatic cervical SCI reported limited neurological benefit [18]. This study inves- tigated the regenerative capabilities of hNSPCs transduced with a GDNF-encoding or mock adenoviral vector (GDNF- and MockhNSPCs, respectively) implanted into the injured spinal cord of adult rats and compared their therapeutic efficiencies for future clinical trials involving SCI patients.

\section{MATERIALS AND METHODS}

\section{Culture of hNSPCs}

Human fetal brain tissue was obtained from a cadaver at 13 weeks of gestation with full parental consent and the approval of the Research Ethics Committee of Yonsei University College of Medicine, Seoul, Korea (Permit No. 4-2003-0078) [18, 31]. All procedures conformed with the guidelines of the National Institutes of Health and the Korean Government. Briefly, hNSPCs isolated from the fetal telencephalon were grown as neurospheres in serum-free DMEM/F12 (Gibco, Grand Island, NY) containing $1 \%(\mathrm{v} / \mathrm{v})$ penicillin/streptomycin (P/S; Gibco), 1\% N-2 Supplement (Gibco), $8 \mu \mathrm{g} / \mathrm{mL}$ heparin (Sigma, St. Louis, MO), $20 \mathrm{ng} / \mathrm{mL}$ fibroblast growth factor-2 (R\&D Systems, Minneapolis, MN), and $10 \mathrm{ng} / \mathrm{mL}$ leukemia inhibitory factor (Sigma). All cultures were maintained in a humidified incubator containing $5 \% \mathrm{CO}_{2}$ and air at $37^{\circ} \mathrm{C}$. Half of the growth medium was replenished every $3 \sim 4$ days.

\section{Construction and transduction of adenoviral vectors}

Human GDNF cDNA was cloned by PCR and inserted into the CAG-pShuttle plasmid. Adenoviral particles were generated using the AdEasy Adenoviral Vector System (Stratagene, La Jolla, CA) [31]. hNSPCs were transduced with a GDNF-encoding adenoviral vector or a null-encoding adenoviral vector at a multiplicity of infection of 40. After 3 days, dissociated single cells were washed twice with Hank's Balanced Salt Solution (Gibco) containing 10 mM HEPES (Gibco; H-H buffer; $\mathrm{pH}$ 7.4) and suspended in $\mathrm{H}-\mathrm{H}$ buffer at a density of $8 \times 10^{4}$ cells $/ \mu \mathrm{L}$ for transplantation.

\section{ELISA}

Mock-hNSPCs and GDNF-hNSPCs were seeded into a 24-well plate at a density of $2.5 \times 10^{5}$ cells/well. After 24 or $72 \mathrm{~h}$, media were collected and cleared by centrifugation at $3000 \mathrm{rpm}$ for $3 \mathrm{~min}$. The levels of human GDNF were measured using a human GDNF DuoSet ELISA Development kit (R\&D systems) according to the manufacturer's instructions.

\section{In vitro neurite outgrowth assay}

SH-SY5Y human neuroblastoma cells were plated into a polyL-lysine-coated 6-well plate containing growth medium (MEM/ 
F12 containing $10 \%$ fetal bovine serum and $1 \% \mathrm{P} / \mathrm{S}$ ) at a density of $1 \times 10^{5}$ cells/well and cultured for $24 \mathrm{~h}$. Cells were washed twice and then incubated with conditioned medium (CM) of MockhNSPCs (Mock-CM) or GDNF-hNSPCs (GDNF-CM) for $24 \mathrm{~h}$. To quantify neurite length, cells were observed under an Olympus IX71 microscope and analyzed with NeuronJ Software. For immunodepletion, GDNF-CM was pre-incubated with $5 \mu \mathrm{g} / \mathrm{mL}$ anti-GDNF (R\&D Systems) or isotype-matched IgG antibody (R\&D Systems) for $30 \mathrm{~min}$ at $37^{\circ} \mathrm{C}$. After $24 \mathrm{~h}$, neurite lengths were measured as described previously [31]. Each data point represents the average of the data of three independent replicates.

\section{Spinal cord injury}

All animal experiments were performed under a protocol approved by the Institutional Animal Care and Use Committee of Yonsei University College of Medicine and in accordance with the Guidelines for the Care and Use of Laboratory Animals issued by the National Institutes of Health. To avoid controversial effects of hormone and gender differences on functional and histological outcomes, adult male rats were only used. Adult male Sprague Dawley rats $(290 \sim 310 \mathrm{~g})$ were housed in groups of $4 \sim 5$ under a 12 $\mathrm{h}$ light-dark cycle. Food and water was provided ad libitum. Traumatic contusive SCI was induced as described previously [32, 33]. Briefly, rats were anesthetized by intraperitoneally injecting a mixture of $97.5 \mathrm{mg} / \mathrm{kg}$ ketamine (Yuhan Co., Seoul, Korea), $4.24 \mathrm{mg} /$ $\mathrm{kg}$ xylazine (Bayern Korea, Seoul, Korea), and $1 \mathrm{mg} / \mathrm{kg}$ acepromazine (Samu Median, Seoul, Korea). Laminectomy was performed at $\mathrm{T}_{9}$ under sterile conditions. The spinal cord was then contused with a NYU weight-drop device. The dorsal surface of the spinal cord was compressed by dropping a $10 \mathrm{~g}$ rod (diameter: $2.5 \mathrm{~mm}$ ) from a height of $50 \mathrm{~mm}$. The surgical site was sutured in layers. To prevent infection, rats were intraperitoneally injected with $50 \mathrm{mg} /$ kg cefazolin (Chong Kun Dang Pharmaceutical Co., Seoul, Korea). The bladder was emptied twice daily.

\section{Cells transplantation}

Animals were randomly assigned to three groups and injected with $12 \mu \mathrm{L}$ GDNF-hNSPCs $\left(8.0 \times 10^{4}\right.$ cells $\left./ \mu \mathrm{L} ; n=32\right)$, MockhNSPCs $\left(8.0 \times 10^{4}\right.$ cells/ $\left.\mu \mathrm{L} ; n=26\right)$, or $\mathrm{H}-\mathrm{H}$ buffer (vehicle, $n=31$ ) at 7 days after SCI. Rats were anesthetized as above and the laminectomy site was re-exposed. Six microliters of cell suspension or buffer was injected $1.5 \mathrm{~mm}$ rostral and caudal to the lesion center, respectively, using a glass micropipette (diameter: $0.3 \mathrm{~mm}$ ). The needle was set at the lesion center along the midline, moved 1.5 $\mathrm{mm}$ rostrally and caudally along the midline, and then inserted 1.0 $\mathrm{mm}$ deep into the spinal cord relative to the dorsal surface. Each injection was performed over $1 \mathrm{~min}$. To prevent leakage of cells through the injection track, the needle was left in position for 1 min after completion of the injection. Animals in all groups were intraperitoneally injected with $10 \mathrm{mg} / \mathrm{kg} / \mathrm{d}$ cyclosporine beginning 1 day before injection and lasting until they were sacrificed.

\section{Histological assessment and stereological quantification}

Adenovirus-infected cells were plated into 8-well chamber slides (Nunc, Roskilde, Denmark) coated with $10 \mathrm{mg} / \mathrm{mL}$ poly-L-lysine (PLL; Sigma) at a density of $8 \times 10^{4}$ cells/well, and differentiated for 7 days in DMEM/F12 containing 1\% P/S and 1\% N-2 Supplement. Immunocytochemical analysis of cultured cells was performed as described previously [18, 31, 34]. For immunohistochemical analysis, animals were deeply anesthetized and perfused transcardially with PIPES buffer (pH 7.4; Sigma) containing 4\% paraformaldehyde at 9 weeks post-transplantation. The spinal cord was subsequently post-fixed in the perfusing solution overnight at $4^{\circ} \mathrm{C}$. Tissues were then cryoprotected in phosphate-buffered saline (PBS) containing $30 \%$ sucrose for $24 \sim 48 \mathrm{~h}$ at $4{ }^{\circ} \mathrm{C}$. The $\mathrm{T}_{8} \sim \mathrm{T}_{10}$ segment of the spinal cord was separated and embedded in O.C.T compound medium (Sakura Finetek, Torrance, CA). Coronal or sagittal cryostat sections of the cord $(16 \mu \mathrm{m}$ thick) were stained with the antibodies listed in Table 1 , as described previously [18, 31]. For the evaluation of the differentiation patterns of engrafted GDNF- and Mock-hNSPCs in the injured spinal cord, one series of parasagittal sections of the spinal cord of rats injected with Mock- or GDNF-hNSPCs ( $n=6$ per group), each separated by 80 $\mu \mathrm{m}$, were analyzed with immunohistochemistry. The number of total human cell-specific marker ${ }^{+}$cells and colocalized cells with human cell- and neural cell type-specific markers were counted in the four representative regions (injury epicenter, spared tissue adjacent to the epicenter, and sequential $1 \mathrm{~mm}$ segments rostral and caudal to the injury epicenter) in each individual series. The number of engrafted cells differentiated into specific neutral cell types is expressed as a percentage of the total number of donor-derived cells in both groups.

The images were acquired using a confocal microscope (LSM700; Carl Zeiss, Oberkochen, Germany) and a BX51 microscope (Olympus, Center Vally, PA). Stereology was conducted using a BX51 microscope and ImageJ software (National Institutes of Health) [35]. Systematic random sampling of the tissue was performed according to stereological principles. Starting sections were chosen at random and every sixth section thereafter was analyzed [14].

Lesion volume, spared tissue volume, and glial scar area were quantified in parasagittal sections of the spinal cord of rats injected with vehicle, Mock-hNSPCs, or GDNF-hNSPCs ( $n=5$ per group) by immunostaining for glial fibrillary acidic protein (GFAP) [14] 
Table 1. Lists of antibodies used for immunostaining and primers used for $\mathrm{qPCR}$

\begin{tabular}{|c|c|c|c|c|c|}
\hline Method & Antibody & Species & Dilution & Company & Cat. no. \\
\hline \multirow{6}{*}{$\begin{array}{l}\text { Immunocytochemistry/ } \\
\text { immunohistochemistry }\end{array}$} & Human nestin & Rabbit & $1 / 200$ & Millipore & ABD69 \\
\hline & GFAP & Rabbit & $1 / 1500$ & DAKO & Z0334 \\
\hline & Tuj1 & Mouse & $1 / 200$ & Covance & MMS-435-P \\
\hline & $\mathrm{O} 4$ & Mouse & $1 / 200$ & Millipore & MAB345 \\
\hline & PDGFR & Rabbit & $1 / 100$ & Santa Cruz & SC-338 \\
\hline & GDNF & Rabbit & $1 / 50$ & Santa Cruz & SC-328 \\
\hline \multirow[t]{12}{*}{ Immunohistochemistry } & Ku80 & Rabbit & $1 / 200$ & Cell Signaling & 2180 \\
\hline & STEM121 & Mouse & $1 / 500$ & Clontech & Y40410 \\
\hline & STEM101 & Mouse & $1 / 100$ & Clontech & Y40400 \\
\hline & Human nuclei & Mouse & $1 / 50$ & Chemicon & MAB1281 \\
\hline & Olig2 & Rabbit & $1 / 200$ & Millipore & Ab9610 \\
\hline & S100B & Goat & $1 / 50$ & R\&D Systems & AF1820 \\
\hline & Neurofilament L & Rabbit & $1 / 1500$ & Chemicon & AB9568 \\
\hline & Neurofilament M & Rabbit & $1 / 1500$ & Chemicon & AB1987 \\
\hline & Neurofilament $\mathrm{H}$ & Rabbit & $1 / 1500$ & Chemicon & AB1991 \\
\hline & MBP & Rabbit & $1 / 400$ & DAKO & A0623 \\
\hline & Schwann/2E & Mouse & $1 / 3000$ & Cosmo Bio & CAG-Gu01-M01AS-A \\
\hline & GABA & Rabbit & $1 / 500$ & Sigma & 2052 \\
\hline Gene (protein) & \multicolumn{2}{|c|}{ Amplicon length (bp) } & \multicolumn{3}{|c|}{ Primer sequence } \\
\hline SCN3A (Nav1.3) & \multicolumn{2}{|c|}{158} & \multicolumn{3}{|c|}{$\begin{array}{l}\mathrm{F}: 5^{\prime} \text {-AACGAAAGACGATCAAGACC-3' } \\
\mathrm{R}: 5^{\prime}-\mathrm{CCAAAGAAACATCAACGATCAG-3'}\end{array}$} \\
\hline SCN9A (Nav1.7) & \multicolumn{2}{|c|}{163} & \multicolumn{3}{|c|}{ F: 5'-GGGAACTTGATCTTTACAGGG-3' } \\
\hline \multirow[t]{2}{*}{$18 \mathrm{~S}$ rRNA } & \multirow{2}{*}{\multicolumn{2}{|c|}{77}} & \multicolumn{3}{|c|}{$\begin{array}{l}\text { R:5-ACTGATAATCCTTCCACATCTG-3' } \\
\text { F:5'-TTGATTAAGTCCCTGCCCTTT-3' }\end{array}$} \\
\hline & & & \multicolumn{3}{|c|}{$\mathrm{R}: 5^{\prime}$-CGATCCGAGGGCCTCACTA-3' } \\
\hline
\end{tabular}

and using the Cavalieri probe at $4 \times$. Lesion volume was quantified as the area of the lesion epicenter that was devoid of GFAP staining using a $90 \mu \mathrm{m}$ grid. Spared tissue volume was quantified in 4 $\mathrm{mm}$ segments rostral and caudal to the injury epicenter, excluding the lesions using a $90 \mu \mathrm{m}$ grid. Glial scar area was quantified by measuring the area of dense GFAP immunostaining near the injury epicenter, excluding the lesion, which was defined as the area lacking GFAP immunostaining, using a $90 \mu \mathrm{m}$ grid $[14,36]$.

Three parasagittal sections ( $n=5$ per group) were collected for immunohistochemistry of GABA and the SC marker Schwann/2E and stereological analysis. The lesion epicenter was captured using a BX51 microscope and the immunostaining densities were measured using ImageJ. To correct for non-specific labeling, the background intensity in a non-immunoreactive area was subtracted from each value. The immunostaining densities of GABA and Schwann/2E were normalized against that in the sham-operated control.

Ten coronal sections ( $n=5$ per group) of the $\mathrm{T}_{8}$ segment were randomly selected and incubated with an anti-NPY antibody followed by a biotinylated antibody (Jackson ImmunoResearch, West Grove, PA). Signals were visualized using a Vectastatin Elite ABC kit (Vector Laboratories, Burlingame, CA) according to the manufacturer's instructions. Images were acquired using a BX51 micro- scope. The optical density of immunostaining was quantified at lamina I of the dorsal horn and normalized against that at lamina VII [37]. The optical density of NPY immunostaining density was normalized against that in the sham-operated control.

Parasagittal sections ( $n=5$ per group) were stained with Luxol Fast Blue (LFB; Sigma) and Cresyl violet (CV; Sigma). Images were acquired using a BX51 microscope. The demyelinated area was measured as the area of CV staining that was devoid of LFB staining. The demyelinated volume was calculated using three sections spaced $90 \mu \mathrm{m}$ using Cavalieris principle and expressed as a percentage of the total cord tissue volume on each slide [38].

\section{Ultrastructural imaging}

Rats were anesthetized as described above and perfused transcardially with cold PBS followed by 2\% PIPES buffer containing 2\% paraformaldehyde and 2\% glutaraldehyde (Merck, Darmstadt, Germany) at 9 weeks post-transplantation. The $\mathrm{T}_{8} \sim \mathrm{T}_{10}$ segment of the spinal cord was separated and post-fixed in the same fixatives overnight at $4{ }^{\circ} \mathrm{C}$. Fixed cord tissues were embedded in 3\% agarose (Sigma) and axially sectioned into $150 \mu \mathrm{m}$-thick slices using a vibratome (Campden Instruments, Loughborough, England). Sections were washed with PBS for 15 min and treated with phosphate buffer containing $1 \%$ osmium tetroxide for $2 \mathrm{~h}$. After 
washing in phosphate buffer, sections were dehydrated in graded ethanol solutions, incubated in propylene oxide (Polysciences, Warrington, PA) for $10 \mathrm{~min}$, and embedded in Epon mixture (Epon ${ }^{\circledR} 812$; Polysciences). Embedded tissue were sectioned into $80 \mathrm{~nm}$-thick slices using a ultramicrotome (Leica Microsystems, Wetzlar, Germany). The slices were placed on a copper grid (Electron Microscopy Sciences, Hatfield, PA), and stained in uranyl acetate (Polysciences) and lead citrate. Images were acquired using a transmission electron microscope (JEM-1011; JEOL, Peabody, MA). The myelin G-ratio was quantified as the ratio of the axon diameter to the diameter of the axon plus the surrounding myelin sheath. The total number of axons sampled in the vehicle-, MockhNSPC-, and GDNF-hNSPC-injected groups ( $n=5$ per group) was 192,172 , and 164 , respectively [39].

\section{Tract tracing studies}

Six weeks after administration of GDNF-hNSPCs, MockhNSPCs, or vehicle ( $n=8$ per group), corticospinal tract (CST) projections were bilaterally traced under anesthesia with biotinylated dextran amine (BDA; 10,000 MW, 10\% in $\mathrm{ddH}_{2} \mathrm{O}$; Invitrogen, Grand Island, NY). A glass micropipette needle attached to a 10 $\mu \mathrm{L}$ Hamilton syringe placed on an infusion pump (KD Scientific, Holliston, MA) was stereotactically guided, and 1.0 $\mu \mathrm{L}$ BDA was bilaterally injected into four sites over the sensorimotor cortex at a depth of $1.5 \mathrm{~mm}$ (Bregma -1 mm, sagittal suture $1.5 \mathrm{~mm}$; Bregma $1 \mathrm{~mm}$, sagittal suture $1.5 \mathrm{~mm}$, respectively). All injections were performed over $1 \mathrm{~min}$, and the injection micropipette was kept in place for an additional $1 \mathrm{~min}$ after completion of the injection to minimize leakage upon withdrawal. Animals were sacrificed 3 weeks later. BDA-labelled fibers were visualized in axial spinal cord sections $(30 \mu \mathrm{m})$ with streptavidin-conjugated Texas Red or 3,3'-diaminobenzidine (DAB; Sigma) and ammonium nickel sulfate using a Vectastatin Elite ABC Kit (Vector Laboratories, Burlingame, $\mathrm{CA}$ ) according to the manufacturer's instructions. The number of $\mathrm{BDA}^{+}$fibers in the dorsal CST (dCST) was determined from images acquired with a confocal microscope using ImageJ software. Axonal fibers in the dCST were evaluated in three axial sections every $1.0 \mathrm{~mm}$ from $6.0 \mathrm{~mm}$ rostral to $6.0 \mathrm{~mm}$ caudal to the lesion epicenter. The number of axonal fibers was normalized against the number of main dCST fibers in the $\mathrm{C}_{1}$ segment of the spinal cord (\% fibers).

In the same groups of rats, the retrograde tracer Fluorogold (FG; $1.0 \mu \mathrm{L}$ of $4 \%$ FG dissolved in $\mathrm{ddH}_{2} \mathrm{O}$; Abcam, Cambridge, MA) was stereotactically injected under anesthesia with a glass micropipette into the left and right sides of the spinal cord at the $\mathrm{L}_{2}$ level at 8 weeks after administration of GDNF-hNSPCs, Mock-hNSPCs, or vehicle. Rats were sacrificed 1 week later. The spinal cords were removed and divided into five segments (cervical, upper thoracic, injury epicenter, lower thoracic, and lumbar parts), and then postfixed, cryoprotected, and frozen in O.C.T compound as described above. FG-labeled propriospinal neurons (PSNs) were stained with an anti-FG antibody and DAB. Sections were mounted with Vectashield mounting medium or Permount (Fisher Scientific, Fair Lawn, NJ) [40]. To analyze the formation of contacts between the cervical dCST and PSNs, BDA-labelled CST collateral fibers were visualized in axial spinal cord sections with DAB and ammonium nickel sulfate, and FG-labeled PSNs were stained with an anti-FG antibody and biotinylated secondary antibody, combined with streptavidin-HRP in the ABC (Avidin Biotin Complex) method. The numbers of collaterals sprouting from the main dCST, propriospinal neurons (PSNs), and contacts between dCST collaterals and PSNs were counted in 20 consecutive axial sections of the cervical enlargement of the spinal cord $\left(\mathrm{C}_{3}\right.$ to $\left.\mathrm{C}_{5}\right)$ at an interval of $150 \mu \mathrm{m}$. To control for variation in the tracing efficiency, the number of collaterals from the dCST was divided by the number of main dCSTs in the same sections. The number of contacts between dCST collaterals and PSNs was also counted in the same sections.

\section{Quantitative real-time reverse transcription-polymerase chain reaction ( $q R T$-PCR)}

At 9 weeks post-transplantation, total RNA was isolated from the $\mathrm{T}_{8} \sim \mathrm{T}_{10}$ segment of the spinal cord in rats injected with GDNF-hNSPCs, Mock-hNSPCs, and vehicle as well as sham-operated controls ( $n=5$ per group) using TRI reagent (Molecular Research Center, Cincinnati, OH). Thereafter, $4 \mu \mathrm{g}$ RNA was reverse-transcribed into cDNA using random primers (Bioneer, Daejeon, Korea) and SuperScript III Reverse Transcriptase (Invitogen). Gene expression was analyzed using SYBR Green on a StepOne Plus thermocycler (Applied Biosystems, Foster City, CA). qRT-PCR was performed using SYBR Green qPCR Master (PCR Biosystems, Wayne, PA) and primers in Table 1, as previously described [36].

\section{Behavior test}

Hindlimb locomotor performance was assessed using the openfield Basso-Beattie-Bresnahan (BBB) scoring system [32]. A rat was placed at the center of a circular open field (diameter: $90 \mathrm{~cm}$, wall height: $7 \mathrm{~cm}$ ) with a non-slip floor for $5 \mathrm{~min}$. Locomotor activity of the hindlimbs was monitored 1 week after injury and weekly for 9 weeks after transplantation. Each score represents a distinct motor functional state from 0 (complete paralysis) to 21 (normal mobility) based on joint movements, stepping ability, coordination, and trunk stability. Examinations were performed by three observers who were unaware of the treatment received 
by each rat. All rats with SCI were block-randomized into four experimental groups (Sham, Vehicle, Mock-hNSPCs, and GDNFhNSPCs; $n=5,24,18$, and 23 , respectively) based on the BBB scores recorded at week 1 to ensure deficits were equivalent across the experimental groups prior to transplantation.

Hindpaw mechanical allodynia was assessed by measuring the withdrawal response to mechanical stimulation with von Frey filaments (Stoelting, Wood Dale, IL) placed on the mid plantar surface of the hindpaw. Before testing, rats were housed in the test cage above a metal mesh and acclimatized for $30 \mathrm{~min}$. The mechanical threshold of a rat was tested prior to surgery and at $14,28,42$, and 56 days after transplantation. The $50 \%$ withdrawal mechanical threshold was measured using Dixon's up-and-down method [41]. A series of von Frey filaments (log unit: 3.61, 3.84, 4.08, 4.31, 4.56, $4.74,4.93$ and 5.18) were serially applied to the hindpaw with an interval of $10 \mathrm{~s}$ in six applications, beginning with the $4.31 \log$ unit von Frey filament.

\section{Statistical analysis}

All statistical analyses were performed using SPSS version 23 (IBM Corp., Armonk, NY, USA). Two groups were compared by the Student's $t$-test. Comparisons of BBB score and 50\% withdrawal threshold among study groups were performed with repeatedmeasures analysis of variance (ANOVA). Where significant differences were observed, one-way ANOVA followed with a Bonferroni post hoc analysis was applied for pairwise comparisons of groups at each time points. Neurite outgrowth, demyelinated volume, g-ratio, tract tracing data, lesion and spared tissue volume, the level of Nav expression, and immunodensity of NPY, GFAP, Schwann/2E, and GABA among study groups were compared by one-way ANOVA, followed by Bonferroni post hoc analysis. Sample sizes were calculated using power analyses with an a-level of 0.05 and a power of 0.8 . Data represent the means \pm standard error of the mean (SEM). $\mathrm{p}<0.05$ was considered statistically significant.

\section{RESULTS}

\section{In vitro characterization of adenovirus-infected hNSPCs}

The morphology, survival, and proliferation rate of GDNF-hNSPCs, Mock-hNSPCs, and hNSPCs were similar under proliferation conditions. To determine whether virus infection increased release of GDNF protein under differentiation conditions, we performed immunocytochemistry with an anti-GDNF antibody and quantified the amount of GDNF protein secreted into the media by performing an ELISA. The concentration of GDNF in CM of GDNF-hNSPCs was $13.30 \pm 0.28 \mathrm{pg} / \mathrm{mL}$ and $22.70 \pm 1.12 \mathrm{pg} / \mathrm{mL}$ after 1 and 3 days, respectively ( $n=3$ per group), while hNSPCs and
Mock-hNSPCs $\left(2.5 \times 10^{5}\right)$ did not express or release GDNF (Fig. 1A and $1 \mathrm{~B})$. Upon culture under differentiation conditions for 7 days, the percentages of cells expressing the early neuronal cell marker neuronal class III $\beta$-tubulin (Tuj1) and the astrocyte marker GFAP were higher and lower, respectively, among GDNF-hNSPCs than among Mock-hNSPCs (Tuj1, 39.4\% $\pm 3.7 \%$ vs. $25.4 \% \pm 3.0 \%$, p $<0.05$; GFAP, $71.7 \% \pm 3.5 \%$ vs. $82.4 \% \pm 1.1 \%$, $\mathrm{p}<0.05 ; n=5$ per group; Fig. $1 \mathrm{C}$ and $1 \mathrm{D})$. In addition, while approximately $67 \%$ and $7 \%$ of MockhNSPC-derived neurons expressed glutamate and GABA, respectively, approximately $35 \%$ and $33 \%$ of GDNF-hNSPC-derived neurons expressed glutamate and GABA, respectively. Some of Mock- and GDNF-hNSPCs differentiated into oligodendrocytes that expressed the oligodendrocyte progenitor cell (OPC) marker O4 (21.8\% $\pm .0 \%$ vs. $25.3 \% \pm 3.0 \%)$ and the oligodendrocyte precursor cell marker PDGFR (19.3\% $\pm 4.5 \%$ vs. $29.5 \pm 6.0 \%)$, although most cells expressed immature cell marker nestin $(87.5 \% \pm 1.6 \%$ vs. $85.0 \% \pm 2.1 \%$; Fig. $1 \mathrm{C}$ and $1 \mathrm{D}$ ). The sum of the percentages of cells expressing each of these markers was higher than 100\%, suggesting that some cells expressed multiple markers. Nestin colocalizes with $\beta$-tubulin III, GFAP, and an oligodendroglial progenitor marker [42]. Additionally, GFAP expression does not always indicate acquisition of an astroglial fate in hNSPCs because immature hNSPCs are also GFAP ${ }^{+}$. These findings demonstrate that GDNFhNSPCs preferentially differentiate into neurons, although most remain immature in vitro.

We investigated whether factors secreted by GDNF-hNSPCs stimulated neurite outgrowth of SH-SY5Y neuroblastoma cells. Both GDNF- and Mock-CM significantly enhanced neurite length compared with that in the control group $(86.1 \pm 3.5 \mu \mathrm{m}$ vs. $38.3 \pm 1.8 \mu \mathrm{m}, \mathrm{p}<0.001$ and $59.0 \pm 2.7 \mu \mathrm{m}$ vs. $38.3 \pm 1.8 \mu \mathrm{m}, \mathrm{p}<0.001$, respectively; Fig. 1E and $1 \mathrm{~F})$. In addition, neurite outgrowth was enhanced more by GDNF-CM than by Mock-CM ( $<<0.01$; Fig. $1 \mathrm{E}$ and $1 \mathrm{~F}$ ). To confirm that GDNF secreted by GDNF-hNSPCs directly affects neurite outgrowth of SH-SY5Y cells, an immunodepletion study was performed. Neurite length of SH-SY5Y cells treated with GDNF-CM pre-incubated with an anti-GDNF antibody was significantly shorter than that of SH-SY5Y cells treated with GDNF-CM pre-incubated with isotype-matched IgG $(62.4 \pm 3.1 \mu \mathrm{m}$ vs. $81.9 \pm 3.4 \mu \mathrm{m} ; \mathrm{p}<0.05$; Fig. $1 \mathrm{E}$ and $1 \mathrm{~F})$. Therefore, GDNF secreted by GDNF-hNSPCs is directly associated with neurite outgrowth.

\section{Engraftment, distribution, and differentiation of GDNF- hNSPCs following transplantation}

Nine weeks after implantation of GDNF-hNSPCs into the injured spinal cord of rats, grafted cells showed robust engraftment, long-term survival, an extensive distribution throughout the in- 
A

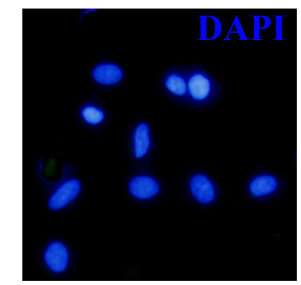

Mock-hNSPCs

D

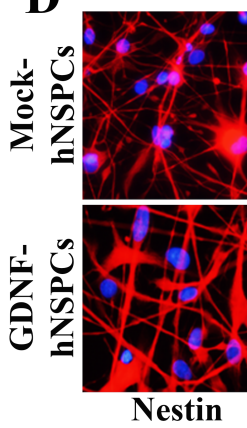

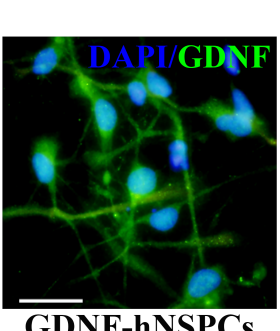

$\overline{\text { GDNF-hNSPCs }}$
B

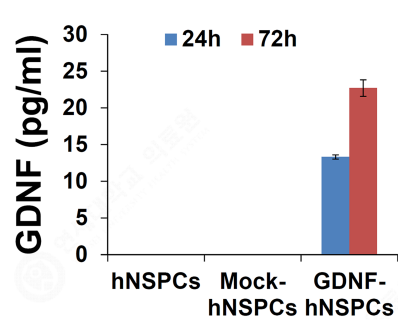

C $\left.{ }^{100}\right] \quad$ Mock-hNSPCS $=$ GDNF-hNSPCS

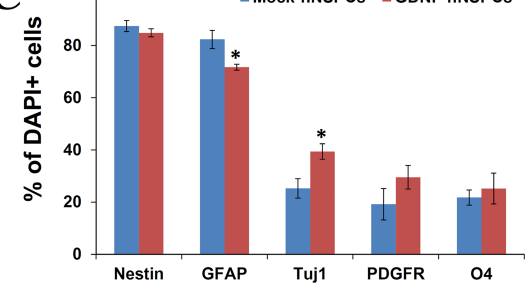

E

F
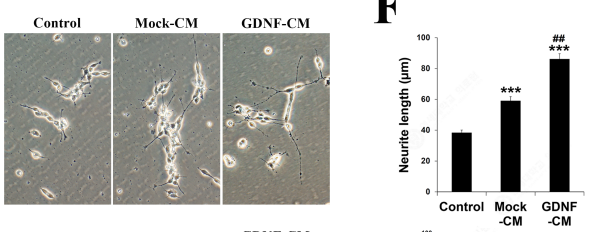

GDNF-CM GDNF-CM GDNF-CM
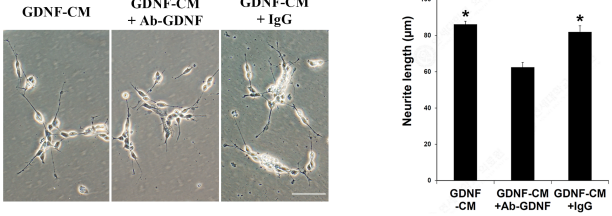

Tuj1

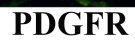

O4
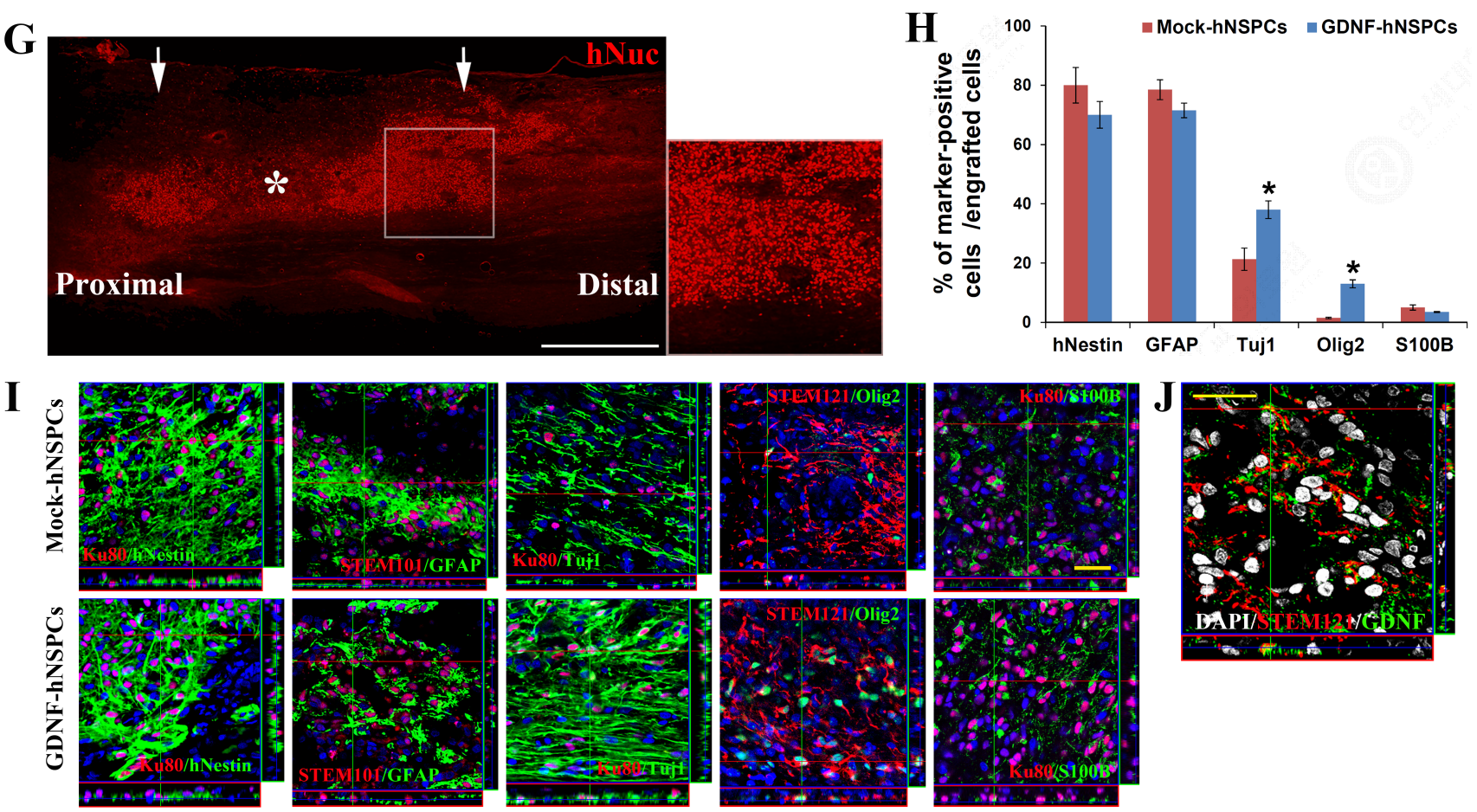

Fig. 1. In vitro and in vivo characterization of GDNF-hNSPCs. (A) Compared with Mock-hNSPCs (DAPI', blue), many of GDNF-hNSPCs express GDNF (green). (B) An ELISA of GDNF in Mock- and GDNF-CM. (C, D) Representative images of Mock- and GDNF-hNSPCs stained for differentiation markers and quantification of the percentages of cells that expressed these markers. DAPI ${ }^{+}$Cells (blue) express nestin (red), GFAP (green), Tuj1 (red), PDGFR (green), and O4 (red). (E, F) Representative images of CM-treated SH-SY5Y cells and quantification of average neurite length under different experimental conditions. (G) hNuc ${ }^{+}$GDNF-hNSPCs (red) implanted into the injured spinal cord show robust engraftment and an extensive distribution throughout the lesion and adjacent areas. The boxed area is shown at high magnification in the right panel. The asterisk indicates the lesion epicenter and arrows indicate the cell transplantation sites rostral and caudal to the lesion epicenter. (H, I) Representative images of Mock- and GDNF-hNSPCs stained for differentiation markers in vivo and quantification of the percentages of cells that expressed these markers. Confocal images show that hNSPCs positive for the human cell markers Ku80, STEM101, and STEM121 colocalize with differentiation markers (green). (J) Engrafted STEM121 ${ }^{+}$ GDNF-hNSPCs (red) express GDNF (green). Scale bars: $50 \mu \mathrm{m}$ in A, D; $100 \mu \mathrm{m}$ in E; $1 \mathrm{~mm}$ in G; and $30 \mu \mathrm{m}$ in I, J. Data represent the means \pm SEM. ${ }^{*} \mathrm{p}<0.05$ vs. Mock-hNSPCs in $\mathrm{C}, \mathrm{H} ;{ }^{*} \mathrm{p}<0.05$ vs. vehicle, ${ }^{* * *} \mathrm{p}<0.001$ vs. vehicle, $\# \# \mathrm{p}<0.01$ vs. Mock-hNSPCs in F. 
jured spinal cord, and integration toward the peri-lesion site (Fig. $1 G)$. The percentages of cells expressing the early neuronal marker Tuj1 and the oligodendroglial progenitor marker Olig2 were significantly higher among grafted GDNF-hNSPCs than among grafted Mock-hNSPCs (TUJ1, 38.4\% $\pm 3.0 \%$ vs. $21.3 \% \pm 3.7 \%$, $\mathrm{p}<0.05$ and Olig2, $13.5 \% \pm 1.3 \%$ vs. $1.5 \% \pm 0.3 \%$, $\mathrm{p}<0.05$, respectively; Fig. 1H and 1I). The majority of grafted GDNF- and MockhNSPCs expressed nestin and GFAP (nestin, $70.2 \% \pm 4.5 \%$ vs. $80.1 \% \pm 6.0 \%$ and GFAP, $71.5 \% \pm 2.4 \%$ vs. $78.5 \% \pm 3.3 \%$, respectively) although the percentages of cells expressing these markers tended
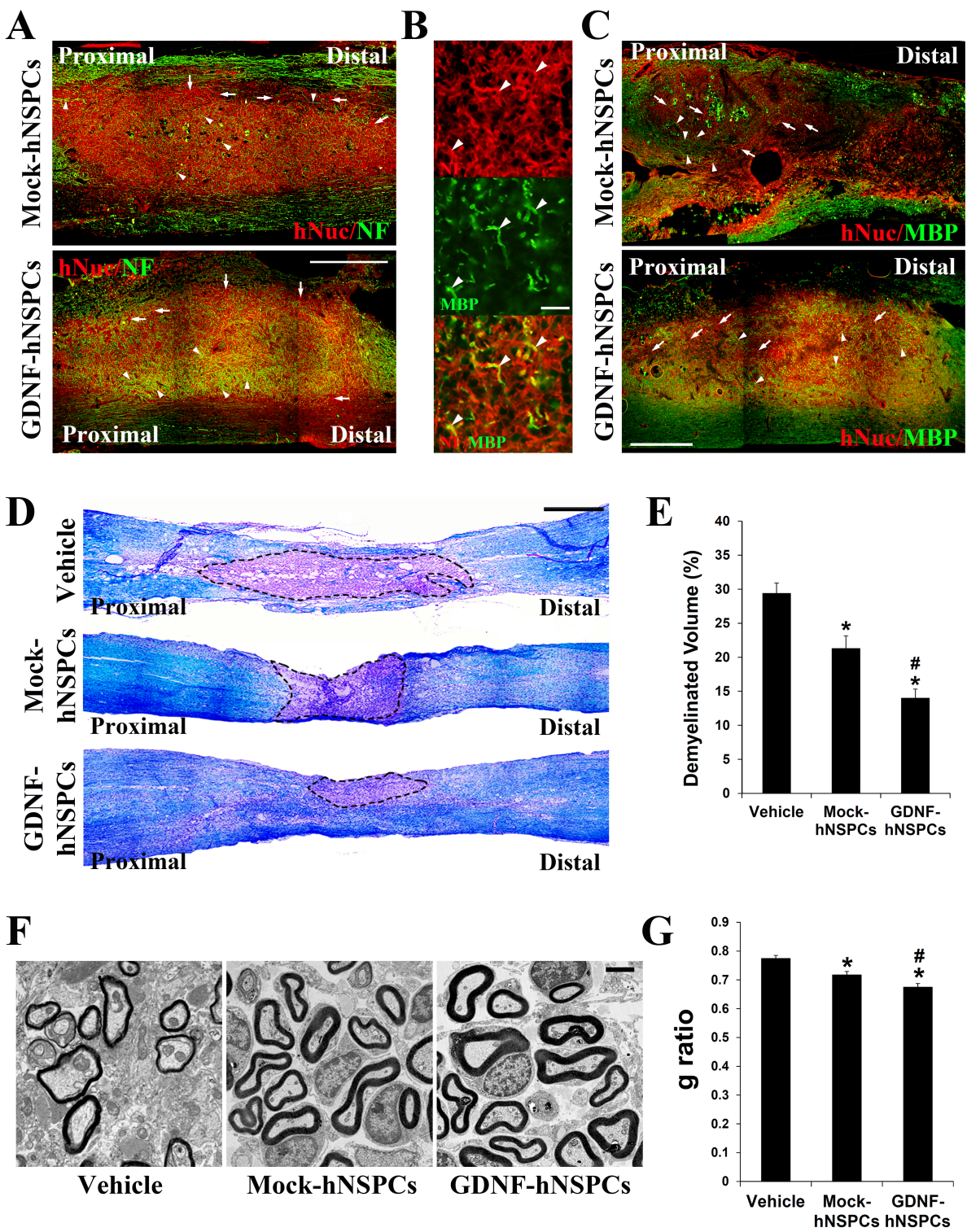

Fig. 2. Grafted GDNF-hNSPCs enhance neurite outgrowth, axonal extension, and myelination in the injured spinal cord. (A) Multiple NF ${ }^{+}$neuronal processes (green) extend over engrafted hNuc human cells (red). Compared with Mock-hNSPCs, GDNF-hNSPCs significantly enhance neurite extension and host axonal sprouting. Arrows indicate $\mathrm{hNuc}^{+}$donor-derived cells (red) and arrowheads indicate $\mathrm{NF}^{+}$neuronal fibers (green). (B) Under high magnification, many $\mathrm{NF}^{+}$neuronal processes (red) are co-labeled with an anti-MBP antibody (green). Arrowheads indicate co-labeled myelinated neuronal fibers (yellow or orange). (C) The intensity of MBP staining in engrafted and adjacent areas is markedly higher in the GDNF-hNSPC-treated group than in the Mock-hNSPC-treated group. Arrows indicate hNuc ${ }^{+}$donor-derived cells (red) and arrowheads indicate $\mathrm{MBP}^{+}$myelinated neuronal fibers (green). (D) Representative images of LFB staining (blue) in the lesion and adjacent areas of the spinal cord. Dashed lines indicate demyelinated areas. (E) Quantification of the demyelinated volume in the different experimental groups. (F, G) Representative electron microscopic images of axially sectioned spinal cords $\left(\mathrm{T}_{8} \sim \mathrm{T}_{10}\right)$ and quantification of G-ratio. $(\mathrm{H}, \mathrm{I})$ Representative images of Schwann/2E immunostaining (green) in the injured spinal cords and quantification of immunostaining density in the different experimental groups. Scale bars: $1 \mathrm{~mm}$ in A, C, D; $100 \mu \mathrm{m}$ in B; $1 \mu \mathrm{m}$ in F, and 500 $\mu \mathrm{m}$ in H. Data represent the means \pm SEM. ${ }^{*} \mathrm{p}<0.05$ vs. vehicle, $\# \mathrm{p}<0.01$ vs. Mock-hNSPCs. 
H

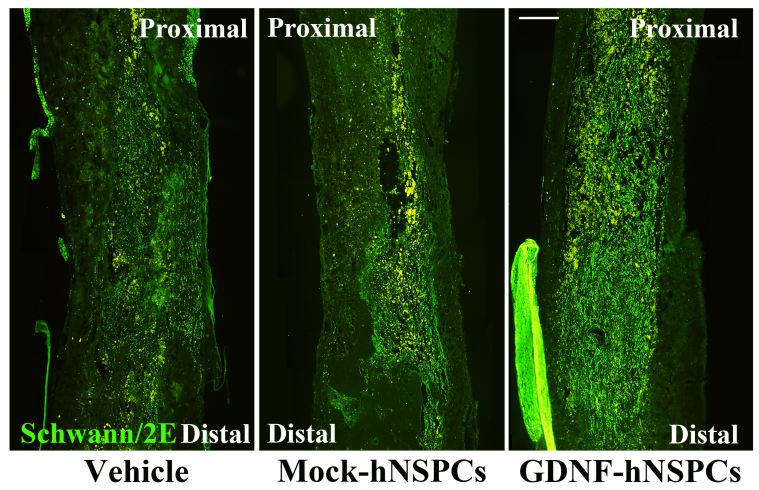

I

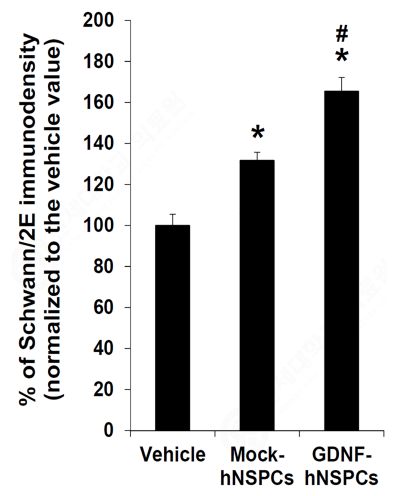

Fig. 2. Continued.

to be lower among GDNF-hNSPCs than among Mock-hNSPCs. Low percentages of grafted GDNF- and Mock-hNSPCs expressed the astrocyte marker $\mathrm{S} 100 \beta(3.5 \% \pm 0.9 \%$ vs. $5.1 \% \pm 0.2 \%$, respectively; Fig. 1H and 1I). Grafted GDNF-hNSPCs also expressed GDNF in the injured spinal cord (Fig. 1J). These data demonstrate that GDNF-hNSPCs implanted into the injured spinal cord show robust engraftment, long-term survival, an extensive distribution, and differentiate into all three CNS neural cell types (preferentially neurons and oligodendrocytes), although most remain immature.

\section{Implantation of GDNF-hNSPCs enhances neurite outgrowth, axonal extension and myelination in the injured spinal cord}

When spinal cords were analyzed at 9 weeks post-transplantation, many hNuc ${ }^{+}$GDNF- and Mock-hNSPCs had survived and migrated extensively to sites rostral and caudal to the lesion epicenter, including spared tissue surrounding the lesion. In addition, multiple neuronal processes expressing NF extended over engrafted human cells (Fig. 2A). Compared with grafted Mock-hNSPCs, grafted GDNF-hNSPCs markedly promoted neurite extension and induced host axonal sprouting (Fig. 2A). These findings suggest that GDNF is one of the most important factors secreted by implanted GDNF-hNSPCs to promote neurite outgrowth and axonal extension in SCI.

High magnification imaging revealed that many $\mathrm{NF}^{+}$neuronal processes were co-labeled with an antibody to the mature oligodendrocyte marker myelin basic protein (MBP) (Fig. 2B). To investigate the effect of GDNF-hNSPCs on axonal myelination after SCI, histological analysis was performed with an anti-MBP antibody and LFB at 9 weeks post-implantation. The intensity of MBP staining in engrafted sites and adjacent areas was markedly higher in the GDNF-hNSPC-treated group than in the Mock-hNSPCtreated group (Fig. 2C). Staining with LFB and CV was performed to label myelinated regions in parasagittal sections of the spinal cord. The blue area indicated myelinated axons, whereas the violet area indicated demyelination of axons. The demyelinated volume percentage (relative to the total tissue volume) in injured and adjacent areas was significantly lower in the Mock-hNSPC- and GDNF-hNSPC-treated groups than in the vehicle-treated group (vehicle, $29.4 \% \pm 1.5 \%$ vs. Mock-hNSPCs, $21.3 \% \pm 1.9 \%, \mathrm{p}<0.05$ and vehicle vs. GDNF-hNSPCs, $14.0 \% \pm 1.4 \%$, p<0.05). Furthermore, the demyelinated volume percentage was significantly lower in the GDNF-hNSPC-treated group than in the Mock-hNSPC-treated group ( $\mathrm{p}<0.05$; Fig. $2 \mathrm{D}$ and $2 \mathrm{E}$ ). To investigate the arrangement of oligodendrocyte-remyelinated axons, the G-ratio was measured using electron microscopy images. The lower the G-ratio, the greater is the extent of remyelination. Conversely, a higher G-ratio indicates thinner myelin sheaths and incomplete remyelination. The G-ratio was significantly lower in the Mock-hNSPC- and GDNF-hNSPC-treated groups than in the vehicle-treated group $(0.72 \pm 0.01$ vs. $0.77 \pm 0.01, \mathrm{p}<0.05$ and $0.67 \pm 0.01$ vs. $0.77 \pm 0.01$, $\mathrm{p}<0.05$, respectively). In addition, the G-ratio was significantly lower in the GDNF-hNSPC-treated group than in the MockhNSPC-treated group ( $\mathrm{p}<0.05$; Fig. $2 \mathrm{~F}$ and $2 \mathrm{G})$. These findings indicate that grafted GDNF-hNSPCs enhance host axonal regeneration and myelination in the injured spinal cord. Moreover, myelin sheaths of regenerated axonal fibers were thicker in the GDNFhNSPC-treated group than in the vehicle- and Mock-hNSPCtreated groups at the ultrastructural level.

To assess SCs after SCI, we measured the immunostaining density of the SC marker Schwann/2E in the injured spinal cord. The immunostaining density were normalized against that in the vehicle-treated group. The normalized Schwann/2E immunostaining density was significantly higher in the GDNF-hNSPC- and Mock-hNSPC-treated groups than in the vehicle-treated group (165.4\% $\pm 6.8 \%$ and $131.7 \% \pm 4.0 \%, \mathrm{p}<0.05$, respectively). Moreover, 
it was significantly higher in the GDNF-hNSPC-treated group than in the Mock-hNSPC-treated group ( $\mathrm{p}<0.05$; Fig. $2 \mathrm{H}$ and $2 \mathrm{I}$ ). Thus, grafted GDNF-hNSPCs substantially facilitate infiltration of SCs into the injured spinal cord and this contributes to myelin repair.
Implantation of GDNF-hNSPCs protects some injured corticospinal fibers, promotes the formation of detour circuits, and reduces lesion volume and glia scar formation

To examine whether GDNF-hNSPCs implanted into the injured spinal cord protect the dCST, BDA was injected into the sensorimotor cortex at 6 weeks post-transplantation. Texas Redlabeled dorsal corticospinal axonal fibers in tissue adjacent to the
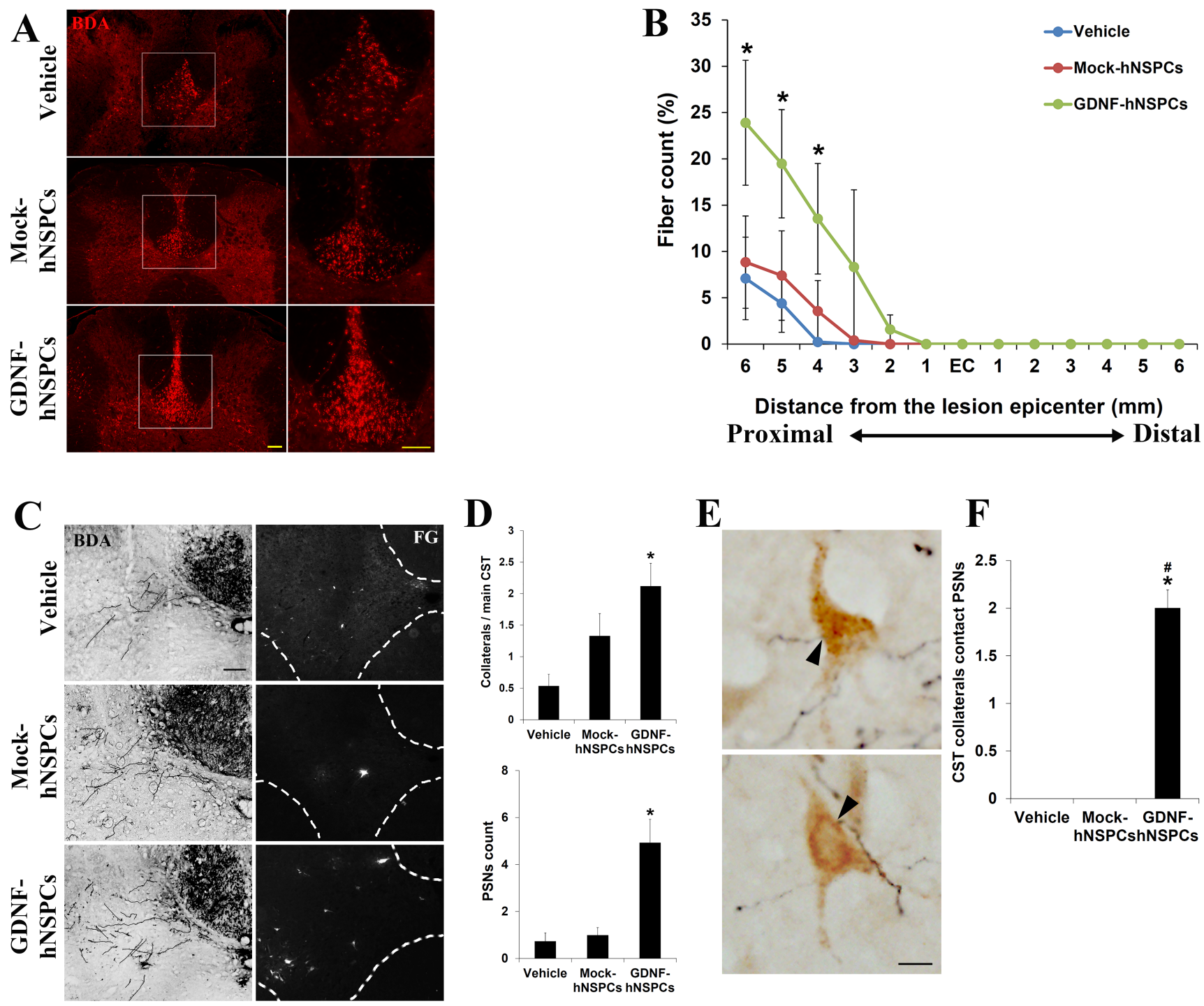

Fig. 3. Grafted GDNF-hNSPCs protect the dCST, promote the formation of detour circuits, and reduce lesion volume and glia scar formation. (A) Representative images of BDA-labeled dCST fibers (red) at $6 \mathrm{~mm}$ rostral to the lesion epicenter in the different experimental groups. The boxed areas in the left panels are shown at high magnification in the right panels. (B) Quantification of the number of dCST fibers up to $6 \mathrm{~mm}$ rostral and caudal to the lesion epicenter. (C) Representative images of dCST collaterals (black, left panel) and FG-labeled PSNs (white, right panel) in the cervical enlargement of the spinal cord in the different experimental groups. The white dashed lines in the right panel indicate the margins between gray and white matter of the spinal cord. (D) Quantification of dCST collaterals and FG-labeled PSNs in the cervical enlargement. (E) Representative images of the contacts between dCST collaterals (black) and PSNs (brown) in the cervical enlargement. Arrowheads show closely located collateral fibers and PSNs. (F) Quantification of the number of contacts between dCST collaterals and PSNs. (G) Representative images of GFAP immunostaining in the lesion and adjacent areas of the spinal cord in the different experimental groups. Dashed lines indicate GFAP-negative areas. (H) Quantification of lesion volume, spared tissue volume, and glial scars. Scale bars: $100 \mu \mathrm{m}$ in A, C; $40 \mu \mathrm{m}$ in E; and $1 \mathrm{~mm}$ in G. Data represent the means \pm SEM. ${ }^{*} \mathrm{p}<0.05$ vs. vehicle, ${ }^{* * *} \mathrm{p}<0.001 \mathrm{vs}$. vehicle, ${ }^{*} \mathrm{p}<0.01$ vs. Mock-hNSPCs. 

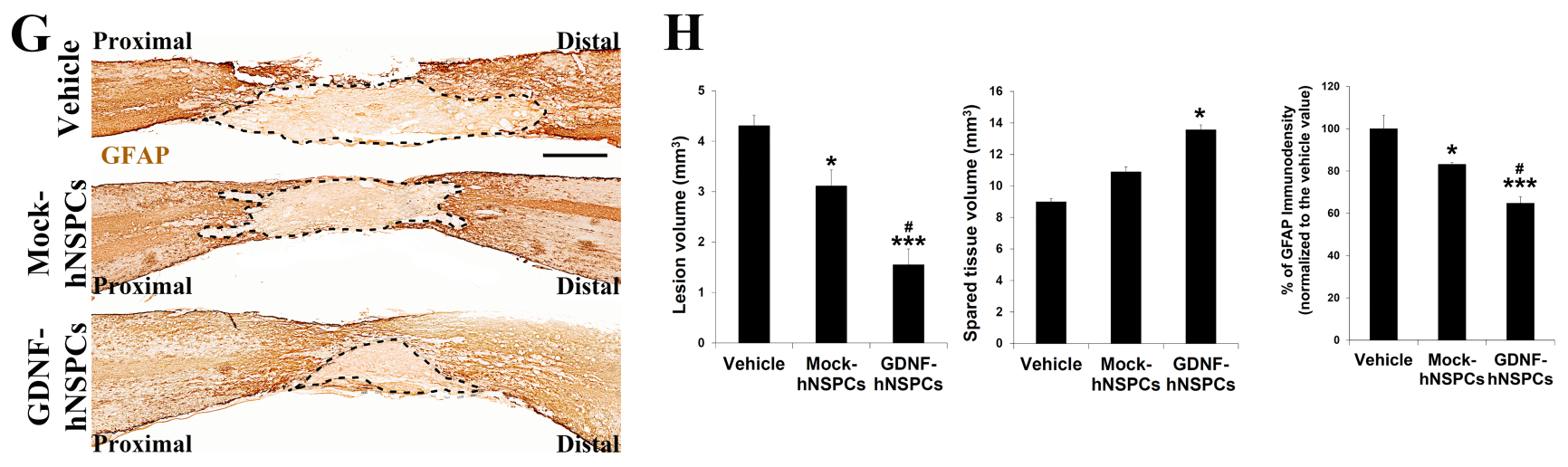

Fig. 3. Continued.

lesion epicenter were detected 3 weeks later. The density of BDAlabeled fibers at $6 \mathrm{~mm}$ rostral to the lesion epicenter was markedly higher in the GDNF-hNSPC-treated group than in the vehicleand Mock-hNSPC-treated groups (Fig. 3A). The number of dCST fibers at 4,5 , and $6 \mathrm{~mm}$ rostral to the lesion epicenter was higher in the GDNF-hNSPC-treated group than in the Mock-hNSPC- and vehicle-treated groups $(\mathrm{p}<0.05$; Fig. 3B). However, BDA-labeled dCST fibers were not detected caudal to the injury epicenter in any group.

To examine whether GDNF-hNSPCs implanted into the injured spinal cord promote the formation of detour circuits in the same group of rats, the retrograde tracer FG was injected into the spinal cord at the $\mathrm{L}_{2}$ level at 8 weeks after injection of GDNF-hNSPCs, Mock-hNSPCs, or vehicle. At 9 weeks post-injection, the numbers of collaterals sprouting from the main dCST, PSNs, and contacts between collaterals and PSNs were counted in the cervical enlargement of the spinal cord. When the number of collaterals from the dCST was divided by the number of main dCSTs in the same sections, the percentage of collaterals was significantly higher in the GDNF-hNSPC-treated group than in the vehicle-treated group $(2.1 \pm 0.4 \%$ vs. $0.5 \pm 0.2 \%, \mathrm{p}<0.05)$. This value tended to be higher in the Mock-hNSPC-treated group than in the vehicle-treated group; however, this difference was not significant ( $1.3 \pm 0.4 \%$ vs. vehicle; Fig. 3C and 3D). The average number of FG-labeled PSNs was significantly higher in the GDNF-hNSPC-treated group than in the vehicle-treated group ( $4.9 \pm 1.0$ vs. $0.7 \pm 0.4, \mathrm{p}<0.05)$. This value tended to be higher in the Mock-hNSPC-treated group than in the vehicle-treated group; however, this difference was not significant (1.0 \pm 0.3 vs. vehicle; Fig. 3C and 3D). Contacts between dCST collaterals and PSNs were observed in the GDNF-hNSPC-treated group, but not in the Mock-hNSPC- and vehicle-treated groups (Fig. 3E and 3F). These results suggest that implantation of GDNFhNSPCs protects some injured corticospinal fibers and promotes the formation of detour circuits in the injured spinal cord.

To examine the neuroprotective effects of GDNF-hNSPC implantation on the injured spinal cord, we assessed the lesion volume, spared tissue volume, and glia scar formation by performing immunostaining for GFAP at 9 weeks post-transplantation. Lesion volume was significantly smaller in the Mock-hNSPC- and GDNF-hNSPC-treated groups than in the vehicle-treated group $\left(3.1 \pm 0.3 \mathrm{~mm}^{3}\right.$ vs. $4.3 \pm 0.2 \mathrm{~mm}^{3}, \mathrm{p}<0.05$ and $1.6 \pm 0.3 \mathrm{~mm}^{3}$ vs. $4.3 \pm 0.2$ $\mathrm{mm}^{3}, \mathrm{p}<0.001$, respectively). Moreover, lesion volume was significantly smaller in the GDNF-hNSPC-treated group than in the Mock-hNSPC-treated group ( $<<0.05$; Fig. $3 \mathrm{G}$ and $3 \mathrm{H})$. Spared tissue volume was significantly larger in the GDNF-hNSPC-treated group than in the vehicle-treated group $\left(13.6 \pm 1.0 \mathrm{~mm}^{3}\right.$ vs. $9.0 \pm 0.7$ $\left.\mathrm{mm}^{3}, \mathrm{p}<0.05\right)$, but did not differ between the vehicle- and MockhNSPC-treated groups (vehicle vs. $10.9 \pm 1.0 \mathrm{~mm}^{3}$; Fig. $3 \mathrm{G}$ and $3 \mathrm{H}$ ). In addition, the normalized GFAP immunostaining density was significantly lower in the GDNF-hNSPC- and Mock-hNSPCtreated groups than in the vehicle-treated group $(64.8 \% \pm 3.1 \%$, $\mathrm{p}<0.001$ and $83.2 \% \pm 1.0 \%, \mathrm{p}<0.05$, respectively) and was significantly lower in the GDNF-hNSPC-treated group than in the Mock-hNSPC-treated group ( $<<0.05$; Fig. $3 \mathrm{G}$ and $3 \mathrm{H}$ ). These results suggest that grafted GDNF-hNSPCs significantly reduce tissue damage and glia scar formation in the lesion epicenter and adjacent areas of the injured spinal cord.

\section{Implantation of GDNF-hNSPCs facilitates motosensory re- covery}

We evaluated the capacity of implanted GDNF-hNSPCs to improve hindlimb locomotor function in rats with SCI using the open-field BBB scoring system. BBB scores were around 21 in all sham groups. Prior to transplantation, BBB scores did not differ among the three groups. BBB scores across different time points before and after cell transplantation were significantly different 

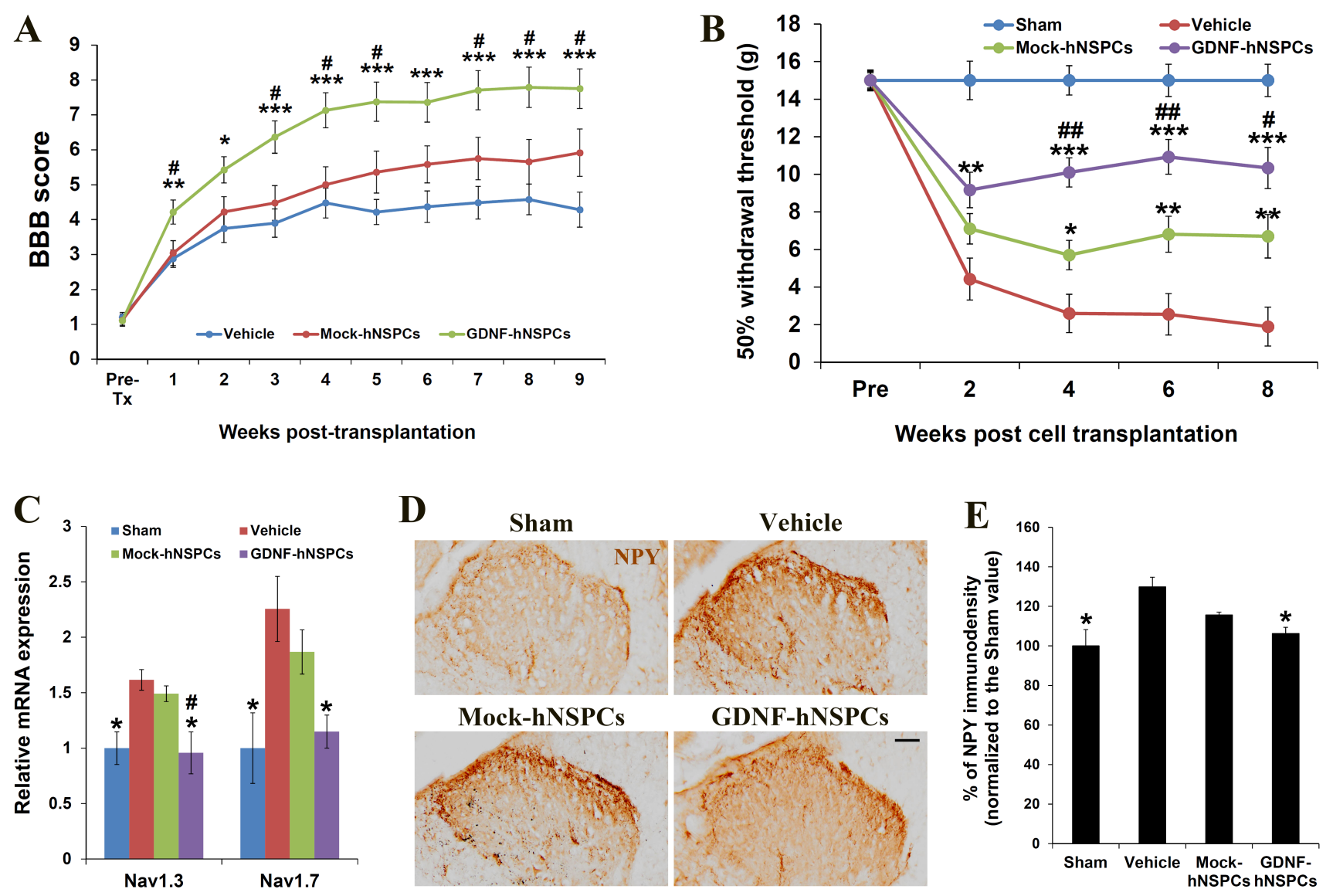

F
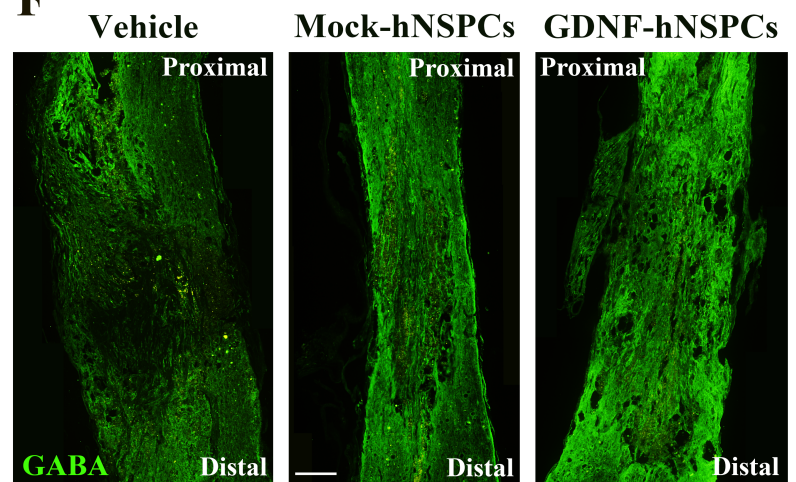

G

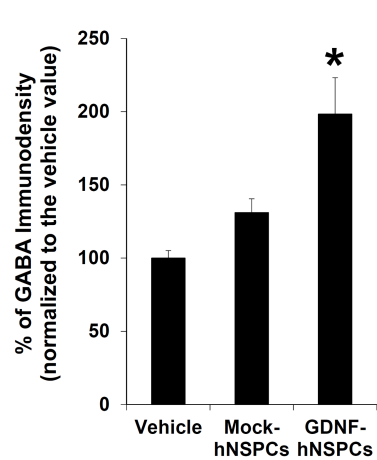

$\mathbf{H}$

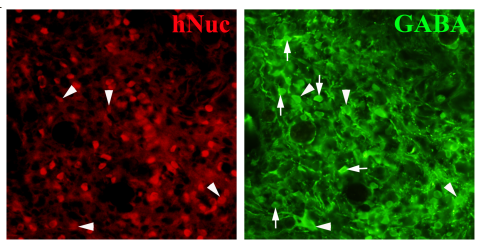

Fig. 4. Transplantation of GDNF-hNSPCs promotes motosensory recovery, reduces expression of voltage-gated sodium channels and NPY, and increases expression of GABA. (A) The BBB open-field walking scores before transplantation and at various time points post-transplantation. (B) Von Frey tests for mechanical allodynia in lesioned hindlimbs of rats with SCI before transplantation and at various time points post-transplantation. (C) qRT-PCR analysis of Nav1.3 and Nav1.9 expression in the $\mathrm{T}_{8} \sim \mathrm{T}_{10}$ segment of the spinal cord. (D, E) Representative images and quantification of NPY expression in lamina I of the dorsal horn in the $\mathrm{T}_{8}$ segment of the spinal cord. (F, G) Representative images and quantification of GABA expression in the $\mathrm{T}_{8} \sim \mathrm{T}_{10}$ segment of the spinal cord. (H) Many engrafted hNuc ${ }^{+}$GDNF-hNSPCs (red, arrowheads) express GABA (green, arrowheads) adjacent endogenous $\mathrm{GABA}^{+}$cells (green, arrows). Scale bars: $200 \mu \mathrm{m}$ in D; $500 \mu \mathrm{m}$ in F; and $50 \mu \mathrm{m}$ in H. Data represent the means \pm SEM. ${ }^{*} \mathrm{p}<0.05 \mathrm{vs}$. vehicle, ${ }^{* *} \mathrm{p}<0.01$ vs. vehicle, ${ }^{* * *} \mathrm{p}<0.001$ vs. vehicle, $\# \mathrm{p}<0.05$ vs. Mock-hNSPCs, \#\#p<0.01 vs. Mock-hNSPCs in A, B. ${ }^{*} \mathrm{p}<0.05$ vs. vehicle, \#p $<0.05$ vs. Mock-hNSPCs in C, E, and $\mathrm{G}$.

among experimental groups ( $\mathrm{p}=0.000)$. GDNF-hNSPCs induced significant functional recovery at 1, 2, 3, 4, 5, 6, 7, 8, and 9 weeks post-transplantation compared with the vehicle control $(4.2 \pm 0.3$ vs. $2.9 \pm 0.3 ; 5.4 \pm 0.4$ vs. $3.8 \pm 0.4 ; 6.4 \pm 0.5$ vs. $3.9 \pm 0.4 ; 7.2 \pm 0.5$ vs. $4.5 \pm 0.4 ; 7.4 \pm 0.6$ vs. $4.2 \pm 0.4 ; 7.4 \pm 0.6$ vs. $4.4 \pm 0.5 ; 7.7 \pm 0.6$ vs. $4.5 \pm 0.5$; $7.8 \pm 0.6$ vs. $4.6 \pm 0.4$; and $7.8 \pm 0.6$ vs. $4.3 \pm 0.5$, respectively; Fig. $4 \mathrm{~A}$ ). 
BBB scores were significantly higher in the GDNF-hNSPC-treated group than in the Mock-hNSPC-treated group at 1, 3, 4, 5, 7, 8, and 9 weeks post-transplantation $(4.2 \pm 0.3$ vs. $3.0 \pm 0.4 ; 6.4 \pm 0.5$ vs. $4.5 \pm 0.5 ; 7.2 \pm 0.5$ vs. $5.0 \pm 0.5 ; 7.4 \pm 0.6$ vs. $5.4 \pm 0.6 ; 7.7 \pm 0.6$ vs. $5.8 \pm 0.6$; $7.8 \pm 0.6$ vs. $5.7 \pm 0.6$; and $7.8 \pm 0.6$ vs. $5.8 \pm 0.5$, respectively; Fig. $4 \mathrm{~A}$ ). $\mathrm{BBB}$ scores tended to be higher in the Mock-hNSPC-treated group than in the vehicle-treated group; however, this difference was not statistically significant. These data demonstrate that implantation of GDNF-hNSPCs improves hindlimb locomotor performance of rats with SCI without causing abnormal neurological behaviors.

We also assessed the effects of GDNF-hNSPC implantation on development of neuropathic pain after SCI by evaluating mechanical allodynia via the von Frey test. The mechanical withdrawal threshold across different time points before and after cell transplantation were significantly different among experimental groups $(\mathrm{p}=0.000)$. The mechanical withdrawal threshold in the vehicletreated group was much lower at 2, 4, 6, and 8 weeks post-injection than prior to injury $(4.4 \pm 0.9,2.6 \pm 0.4,2.6 \pm 0.3$, and $1.9 \pm 0.4$, respectively; Fig. 4B). The mechanical withdrawal threshold was significantly higher in the Mock-hNSPC-treated group than in the vehicle-treated group at 4,6 , and 8 weeks post-transplantation $(5.7 \pm 1.2$ vs. vehicle; $6.8 \pm 1.4$ vs. vehicle; and $6.7 \pm 1.1$ vs. vehicle, respectively; Fig. 4B). The mechanical withdrawal threshold was significantly higher in the GDNF-hNSPC-treated group than in the vehicletreated group at 2, 4, 6, and 8 weeks post-transplantation $(9.2 \pm 1.0$ vs. vehicle,; $10.1 \pm 0.8$ vs. vehicle; $10.9 \pm 0.9$ vs. vehicle; and $10.3 \pm 1.1$ vs. vehicle, respectively). Furthermore, the mechanical withdrawal threshold was significantly higher in the GDNF-hNSPC-treated group than in the Mock-hNSPC-treated group at 4,6, and 8 weeks post-transplantation (Fig. 4B). In the sham group, the mechanical withdrawal threshold was $15 \pm 0.2$. Hence, transplantation of GDNF-hNSPCs efficiently attenuates mechanical allodynia after SCI.

\section{Implantation of GDNF-hNSPCs reverses the upregulation of voltage-gated sodium channels and NPY in the dorsal horn and increase GABA expression after SCI}

To investigate the effect of GDNF-hNSPC implantation on expression of voltage-gate sodium channels (Nav) linked with neuropathic pain in SCI, we analyzed Nav1.3 and Nav1.9 expression in the $\mathrm{T}_{8} \sim \mathrm{T}_{10}$ segment of the spinal cord at 9 weeks post-transplantation by performing qRT-PCR. Expression of Nav1.3 (1.61 \pm 0.09 fold, $\mathrm{p}<0.05)$ and Nav1.7 (2.25 \pm 0.29 -fold, $\mathrm{p}<0.05)$ was higher in the vehicle-treated group than in the sham-operated control. Expression of Nav1.3 (1.45 \pm 0.07 -fold) and Nav1.7 (1.86 \pm 0.20 -fold) tended to be lower in the Mock-hNSPC-treated group than in the vehicle-treated group; however, these differences were not signifi- cant. However, expression of Nav1.3 (0.95 \pm 0.19 -fold, $\mathrm{p}<0.05)$ and Nav1.7 $(1.15 \pm 0.15$-fold, $\mathrm{p}<0.05)$ in the GDNF-hNSPC-treated group was comparable with that in the sham-operated control and was significantly lower than that in the vehicle-treated group (Fig. 4C). In addition, expression of Nav1.3 was significantly lower in the GDNF-hNSPC-treated group than in the Mock-hNSPCtreated group.

The normalized NPY immunostaining density at lamina I of the dorsal horn in the $\mathrm{T}_{8}$ segment of the spinal cord was significantly higher at 9 weeks after vehicle injection $(129.85 \% \pm 4.89 \%, \mathrm{p}<0.05)$ than in the sham-operated control. However, the normalized NPY immunostaining density in the GDNF-hNSPC-treated group $(106.28 \% \pm 3.14 \%, \mathrm{p}<0.05)$ was similar to that in the sham-operated control and was significantly lower than that in the vehicletreated group. The normalized NPY immunostaining density tended to be lower in the Mock-hNSPC-treated group than in the vehicle-treated group; however, this difference was not significant (115.69\% $\pm 1.37 \%$; Fig. $4 \mathrm{D}$ and $4 \mathrm{E})$.

The normalized GABA immunostaining density in the $\mathrm{T}_{8} \sim \mathrm{T}_{10}$ segment of the spinal cord at 9 weeks post-transplantation was significantly higher in the GDNF-hNSPC-treated group (198.36 $\pm 24.81 \%)$ than in the vehicle-treated group and tended to be higher in the Mock-hNSPC-treated group than in the vehicle-treated group; however, this difference was not significant (131.04 $\pm 9.48 \%$; Fig. 4F and 4G). Histochemical analysis confirmed that many implanted GDNF-hNSPCs expressed GABA and thus gave rise to GABAergic cells in the injured spinal cord (Fig. 4H). These results suggest that reversion of Nav and NPY upregulation and increased GABA expression upon implantation of GDNFhNSPCs alleviates mechanical allodynia after SCI.

\section{DISCUSSION}

SCI is a devastating, life-changing disorder with many serious consequences and no known effective treatment. Cell transplantation has emerged as a promising therapeutic method to enhance repair following SCI. Various types of cells, including SCs, oligodendrocyte precursor cells, NSPCs, MSCs, and olfactory ensheathing cells, have been evaluated in terms of their capacity to regenerate the injured spinal cord upon transplantation [43]. Although cell transplantation has been intensely studied, it remains unclear how implanted cells yield therapeutic effects in SCI. We previously reported that human fetal brain-derived primary NSPCs implanted into the injured spinal cord of adult rats exhibit robust engraftment, extensive migration, long-term survival, and differentiation into neuronal and glial cells, although most remain immature, resulting in modest improvement of motosensory function $[18,44]$. 
Following implantation, hNSPCs are suggested to have functional benefits for SCI via a variety of mechanisms, including neuroprotection, neural plasticity, immunomodulation, angiogenesis, neurite outgrowth, axonal extension, and cellular replacement $[18,44]$. Based on these preliminary data, several early-phase clinical trials were performed. These demonstrated that transplantation of human fetal brain- or spinal cord-derived NSPCs is generally feasible and safe, and yields minimal or modest neurological improvement; however, the long-term efficacy and safety remain unproven [18-20]. The patterns of cellular responses to SCI are extremely heterogeneous and complex; therefore, simply delivering specific neural cell types into the injured spinal cord via cell or stem cell therapy is ineffective [44]. Thus, the therapeutic efficiency of hNSPCs for SCI is insufficient and there is no cure for this condition.

One possible treatment strategy for SCI is to prevent neurodegeneration and stimulate regeneration using GDNF. GDNF has extensive effects on repair after SCI and these have been broadly examined. To improve the therapeutic efficiency of GDNF, future research should focus on enhanced delivery methods using biomaterials, nanoparticles, viral vectors, or genetically modified cells to secrete both GDNF and other trophic factors over time [29, 30, 44]. Previous research indicated that direct in vivo GDNF gene therapy using viral vectors or plasmid DNA protects motor neurons and promotes functional recovery after SCI [45]. Adenovirusor herpes simplex virus-mediated GDNF gene therapy reduces motor neuron injury, preserves neuronal fibers, and improves locomotor function in rats with SCI [46-49]. However, in vivo gene therapy using viral vectors is associated with several complications, including non-specific targeting and gene expression, gene silencing, and an immune response to the vector [50].

Ex vivo gene therapy allows the genetically engineered cell product to be characterized in vitro prior to administration and does not involve direct exposure of the patient to the viral vector. In addition, grafted genetically modified cells not only replace the neural cell types lost to injury and support diseased neurons and/ or dysfunctional glia, but also produce additional endogenous trophic and immunomodulatory molecules that augment their therapeutic potential. Various cell types have been used in combined cell and gene therapeutic approaches to provide GDNF to the injured spinal cord [29, 30]. GDNF-overexpressing SCs implanted into the injured spinal cord modulate astrocytic glial scars, promote propriospinal axonal regeneration and synapse formation, and improve functional recovery [51]. Implantation of hydrogel scaffolds containing GDNF-overexpressing SCs into the transected spinal cord also promotes axonal growth and axon myelination [52]. Grafted genetically modified GDNF-secreting MSCs or umbilical cord blood-derived mononuclear cells induce functional recovery and tissue sparing, and increase myelination after SCI [45, 53]. These studies suggest that ex vivo GDNF gene therapy with multiple cell types has beneficial effects for spinal cord repair. This may be because the complicated and dynamic milieu resulting from SCI seems to require combinational treatment approaches. However, while immortalized mouse NSCs constitutively secrete GDNF together with other neurotrophic factors and promote axonal growth after SCI, implantation of mouse NSCs genetically engineered to overexpress GDNF into the injured spinal cord does not improve locomotor function and significant forelimb thermal and mechanical allodynia because these cells exclusively undergo astrocytic differentiation $[9,54]$.

Human fetal cortex-derived primary NSPCs genetically modified to stably express GDNF using a lentivirus survive, migrate, secrete GDNF, and protect degenerating neurons $[55,56]$. These cells are safe and non-tumorigenic, and are currently being used in a phase $1 / 2$ a clinical trial incorporating cell and gene therapy to protect motor neurons in patients with amyotrophic lateral sclerosis [57]. Therefore, in this study, we evaluated the regenerative ability of human fetal brain-derived NSPCs engineered to secrete GDNF via infection of an adenovirus in rats with SCI and compared their therapeutic efficiency with that of Mock-hNSPCs for a future clinical trial. Ex vivo transduction of hNSPCs with a GDNF-encoding adenoviral vector leads to effective expression of GDNF. In addition, GDNF-hNSPCs might secrete multiple other active factors that enhance neuronal survival and neuroplasticity, reduce secondary damage, and stimulate the endogenous repair system via inducing favorable changes in the environmental milieu of the damaged spinal cord $[31,44]$. In the present study, although GDNF-hNSPCs were implanted into the injured spinal cord at 7 days after SCI during the subacute stage, they showed robust engraftment, long-term survival, an extensive distribution, and increased differentiation into neurons and oligodendroglial cells in the lesion and adjacent sites. Therefore, grafted cell-derived GDNF and other therapeutic molecules diffuse beyond the engraftment sites over a long period, which may contribute to extensive neuroprotection, decreased lesion volume, promotion of neurite outgrowth and axonal extension, increased SC migration and myelination, modulation of astrocytic glial scars, and improved motosensory function post-SCI.

Compared with grafted Mock-hNSPCs, grafted GDNF-hNSPCs showed increased differentiation into neurons and oligodendrocytes, although most remained immature, and these cells might be able to reconstitute the injured spinal cord. However, further studies are needed to electrophysiologically confirm the functional neural connections and efficient axonal connectivity between host and donor-derived neurons. 
SCI induces neuronal death at the injury site and extensive axonal dieback of the dCST, which is pivotal for voluntary movement [58]. Neuroprotection of damaged axons and increased axonal plasticity are important for locomotor recovery. hNSPCs produce various neurotrophic factors that support neuroprotection and neurite outgrowth [31]. In addition, GDNF promotes axonal plasticity in CSTs and newly formed PSNs after SCI [59, 60]. After SCI, transected mid-thoracic CST axons sprouted into the cervical gray matter where they contact descending 'long' and 'short' PSNs. Long PSNs originate in the cervical enlargement $\left(\mathrm{C}_{3} \sim \mathrm{C}_{5}\right)$, travel in the ventral and lateral funiculi, and terminate in the ventral horn of the lumbosacral enlargement bridging spinal levels above and below the lesion. In contrast, short PSNs connect the upper $\left(\mathrm{C}_{3} \sim \mathrm{C}_{4}\right)$ and lower $\left(\mathrm{C}_{6} \sim \mathrm{T}_{1}\right)$ cervical cord, not bridging spinal levels above and below the lesion. Transected CST axons sprout into short and long PSNs in the cervical enlargement equally, but sprouts that contacted long PSNs are maintained, thereby creating a new intraspinal circuit (detour circuit) [40]. In this study, transplanted GDNF-hNSPCs induced host axonal regrowth, protected the dCST, and increased the number of PSNs and contacts between dCST collaterals and PSNs in the cervical enlargement promoting the formation of detour circuits in the injured spinal cord.

In terms of motor function recovery after SCI, a previous study reported that grafted GDNF-secreting olfactory ensheathing cells and GDNF-secreting SCs increased the BBB score by almost 8 and about 5 compared with controls, respectively [30]. In this study, the BBB score was approximately 3.5 higher in the group transplanted with GDNF-hNSPCs during the subacute stage after severe contusive SCI than in the control group at 9 weeks post-transplantation. However, it is difficult to directly compare the improvements of BBB scores between studies because the type of SCI (contusion, hemisection, and complete transection), level of injury, concentration of secreted GDNF, GDNF delivery method, and timing of transplantation after SCI differ.

In animal models, GDNF exerts potent analgesic effects on neuropathic pain, although the underlying mechanisms have not been completely elucidated [61]. GDNF reverses nerve injury-induced changes, including expression of the $\mathrm{P} 2 \mathrm{X} 3$ purinoreceptor, sodium channels, neuropeptides, and regulator of G-protein signaling protein 4, as well as IB4 binding, in dorsal root ganglion neurons [61]. However, effective GDNF delivery methods to relieve neuropathic pain are still under investigation. In addition, GDNF-hNSPCs were more differentiated into GABA+ neurons than MockhNSPCs. Thus, the increased supply of donor-derived GABAergic neurons may be able to restore the inhibitory function to the spinal cord dorsal horn to decrease the hyperexcitability that develops in dorsal horn projection neurons [62-65]. The increased GABA production is a potential mechanism of behavioral improvement in the allodynic rat after SCI. Furthermore, GABA as well as other trophic factors including GDNF secreted by transplanted cells would protect endogenous spinal cord inhibitory interneurons after SCI. Thus, not only Mock-hNSPCs but also GDNF-hNSPCs implanted into SCI would attenuate neuropathic pain. Taken together, grafted GDNF-hNSPCs reversed the upregulation of Nav and NPY, increased GABA expression, and a rare differentiation of donor-derived cells into astrocytes, which contributed to marked alleviation of mechanical allodynia after SCI.

Demyelination is a major hurdle for recovery after SCI. Although resident OPCs can replace damaged oligodendrocytes, the inhibitory milieu of the injured spinal cord prevents mature myelination [66]. Transplantation of OPCs or SCs is effective for SCI recovery $[67,68]$. However, OPCs and SCs are difficult to isolate and this limits their use. In this study, grafted GDNF-hNSPCs showed increased differentiation into oligodendrocytes and enhanced migration of SCs toward the injured spinal cord. SCs migrate to lesion sites in the spinal cord, participate in remyelination in these areas, and support axonal growth [45]. GDNF stimulates migration of SCs and enhances myelination through the NCAM signaling pathways that regulate myelin formation [69]. Thus, the combinational approach using hNSPCs and GDNF induced robust remyelination in damaged areas by host SCs and donor-derived oligodendrocytes after SCI.

In summary, transplantation of GDNF-hNSPCs is a promising combinational treatment approach to enhance repair of the injured spinal cord. The GDNF gene was efficiently transduced using an adenoviral vector, and GDNF-hNSPCs expressed GDNF for a long duration both in vitro and in vivo. GDNF-hNSPCs implanted into the injured spinal cord showed robust engraftment, long-term survival, an extensive distribution, and increased differentiation into neurons and oligodendrocytes in the lesion and adjacent areas. This resulted in extensive neuroprotection, decreased lesion volume, promotion of neurite outgrowth and axonal extension, increased SC migration and myelination, modulation of astrocytic glial scars, and improved functional recovery post-SCI. These findings support the investigation of GDNF-hNSPCs in a clinical trial for SCI.

\section{ACKNOWLEDGEMENTS}

This study was supported by grants from Korean Health Technology R\&D Project (HI16C1089) and the Bio \& Medical Technology Development Program of the National Research Foundation (NRF) funded by the Korean government (MSIT) (No. NRF2019M3A9H1032791). 


\section{REFERENCES}

1. Singh A, Tetreault L, Kalsi-Ryan S, Nouri A, Fehlings MG (2014) Global prevalence and incidence of traumatic spinal cord injury. Clin Epidemiol 6:309-331.

2. Thuret S, Moon LD, Gage FH (2006) Therapeutic interventions after spinal cord injury. Nat Rev Neurosci 7:628-643.

3. Hagen EM, Rekand T (2015) Management of neuropathic pain associated with spinal cord injury. Pain Ther 4:51-65.

4. Barnabé-Heider F, Frisén J (2008) Stem cells for spinal cord repair. Cell Stem Cell 3:16-24.

5. Gage FH, Temple S (2013) Neural stem cells: generating and regenerating the brain. Neuron 80:588-601.

6. Hofstetter CP, Holmström NA, Lilja JA, Schweinhardt P, Hao J, Spenger C, Wiesenfeld-Hallin Z, Kurpad SN, Frisén J, Olson L (2005) Allodynia limits the usefulness of intraspinal neural stem cell grafts; directed differentiation improves outcome. Nat Neurosci 8:346-353.

7. Karimi-Abdolrezaee S, Eftekharpour E, Wang J, Morshead CM, Fehlings MG (2006) Delayed transplantation of adult neural precursor cells promotes remyelination and functional neurological recovery after spinal cord injury. J Neurosci 26:3377-3389

8. Keirstead HS, Nistor G, Bernal G, Totoiu M, Cloutier F, Sharp K, Steward O (2005) Human embryonic stem cell-derived oligodendrocyte progenitor cell transplants remyelinate and restore locomotion after spinal cord injury. J Neurosci 25:4694-4705.

9. Lu P, Jones LL, Snyder EY, Tuszynski MH (2003) Neural stem cells constitutively secrete neurotrophic factors and promote extensive host axonal growth after spinal cord injury. Exp Neurol 181:115-129.

10. Teng YD, Lavik EB, Qu X, Park KI, Ourednik J, Zurakowski D, Langer R, Snyder EY (2002) Functional recovery following traumatic spinal cord injury mediated by a unique polymer scaffold seeded with neural stem cells. Proc Natl Acad Sci U S A 99:3024-3029.

11. Ziv Y, Avidan H, Pluchino S, Martino G, Schwartz M (2006) Synergy between immune cells and adult neural stem/progenitor cells promotes functional recovery from spinal cord injury. Proc Natl Acad Sci U S A 103:13174-13179.

12. Hwang DH, Park HH, Shin HY, Cui Y, Kim BG (2018) Insulin-like growth factor-1 receptor dictates beneficial effects of treadmill training by regulating survival and migration of neural stem cell grafts in the injured spinal cord. Exp Neurobiol 27:489-507.

13. Cummings BJ, Uchida N, Tamaki SJ, Salazar DL, Hooshmand
M, Summers R, Gage FH, Anderson AJ (2005) Human neural stem cells differentiate and promote locomotor recovery in spinal cord-injured mice. Proc Natl Acad Sci U S A 102:14069-14074.

14. Salazar DL, Uchida N, Hamers FP, Cummings BJ, Anderson AJ (2010) Human neural stem cells differentiate and promote locomotor recovery in an early chronic spinal cord injury NOD-scid mouse model. PLoS One 5:e12272.

15. Hooshmand MJ, Sontag CJ, Uchida N, Tamaki S, Anderson AJ, Cummings BJ (2009) Analysis of host-mediated repair mechanisms after human CNS-stem cell transplantation for spinal cord injury: correlation of engraftment with recovery. PLoS One 4:e5871.

16. Yan J, Xu L, Welsh AM, Hatfield G, Hazel T, Johe K, Koliatsos VE (2007) Extensive neuronal differentiation of human neural stem cell grafts in adult rat spinal cord. PLoS Med 4:e39.

17. Rosenzweig ES, Brock JH, Lu P, Kumamaru H, Salegio EA, Kadoya K, Weber JL, Liang JJ, Moseanko R, Hawbecker S, Huie JR, Havton LA, Nout-Lomas YS, Ferguson AR, Beattie MS, Bresnahan JC, Tuszynski MH (2018) Restorative effects of human neural stem cell grafts on the primate spinal cord. Nat Med 24:484-490

18. Shin JC, Kim KN, Yoo J, Kim IS, Yun S, Lee H, Jung K, Hwang K, Kim M, Lee IS, Shin JE, Park KI (2015) Clinical trial of human fetal brain-derived neural stem/progenitor cell transplantation in patients with traumatic cervical spinal cord injury. Neural Plast 2015:630932.

19. Ghobrial GM, Anderson KD, Dididze M, Martinez-Barrizonte J, Sunn GH, Gant KL, Levi AD (2017) Human neural stem cell transplantation in chronic cervical spinal cord injury: functional outcomes at 12 months in a phase II clinical trial. Neurosurgery 64(CN_suppl_1):87-91.

20. Curtis E, Martin JR, Gabel B, Sidhu N, Rzesiewicz TK, Mandeville R, Van Gorp S, Leerink M, Tadokoro T, Marsala S, Jamieson C, Marsala M, Ciacci JD (2018) A first-in-human, phase I study of neural stem cell transplantation for chronic spinal cord injury. Cell Stem Cell 22:941-950.e6.

21. Lindvall O, Kokaia Z (2010) Stem cells in human neurodegenerative disorders--time for clinical translation? J Clin Invest 120:29-40.

22. Goldman SA (2016) Stem and progenitor cell-based therapy of the central nervous system: hopes, hype, and wishful thinking. Cell Stem Cell 18:174-188.

23. Airaksinen MS, Saarma M (2002) The GDNF family: signalling, biological functions and therapeutic value. Nat Rev Neurosci 3:383-394.

24. Iannotti C, Ping Zhang Y, Shields CB, Han Y, Burke DA, Xu 
XM (2004) A neuroprotective role of glial cell line-derived neurotrophic factor following moderate spinal cord contusion injury. Exp Neurol 189:317-332.

25. Cheng H, Wu JP, Tzeng SF (2002) Neuroprotection of glial cell line-derived neurotrophic factor in damaged spinal cords following contusive injury. J Neurosci Res 69:397-405.

26. Li L, Wu W, Lin LF, Lei M, Oppenheim RW, Houenou LJ (1995) Rescue of adult mouse motoneurons from injury-induced cell death by glial cell line-derived neurotrophic factor. Proc Natl Acad Sci U S A 92:9771-9775.

27. Watabe K, Ohashi T, Sakamoto T, Kawazoe Y, Takeshima T, Oyanagi K, Inoue K, Eto Y, Kim SU (2000) Rescue of lesioned adult rat spinal motoneurons by adenoviral gene transfer of glial cell line-derived neurotrophic factor. J Neurosci Res 60:511-519.

28. Ramer MS, Bradbury EJ, Michael GJ, Lever IJ, McMahon SB (2003) Glial cell line-derived neurotrophic factor increases calcitonin gene-related peptide immunoreactivity in sensory and motoneurons in vivo. Eur J Neurosci 18:2713-2721.

29. Walker MJ, Xu XM (2018) History of glial cell line-derived neurotrophic factor (GDNF) and its use for spinal cord injury repair. Brain Sci 8:E109.

30. Rosich K, Hanna BF, Ibrahim RK, Hellenbrand DJ, Hanna A (2017) The effects of glial cell line-derived neurotrophic factor after spinal cord injury. J Neurotrauma 34:3311-3325.

31. Lee IS, Koo KY, Jung K, Kim M, Kim IS, Hwang K, Yun S, Lee H, Shin JE, Park KI (2017) Neurogenin-2-transduced human neural progenitor cells attenuate neonatal hypoxic-ischemic brain injury. Transl Res 183:121-136.e9.

32. Basso DM, Beattie MS, Bresnahan JC (1996) Graded histological and locomotor outcomes after spinal cord contusion using the NYU weight-drop device versus transection. Exp Neurol 139:244-256.

33. Kim JH, Kim JY, Mun CH, Suh M, Lee JE (2017) Agmatine modulates the phenotype of macrophage acute phase after spinal cord injury in rats. Exp Neurobiol 26:278-286.

34. Ha S, Jeong SH, Yi K, Chu JJ, Kim S, Kim EK, Yu SW (2019) Autophagy mediates astrogenesis in adult hippocampal neural stem cells. Exp Neurobiol 28:229-246.

35. Pool M, Thiemann J, Bar-Or A, Fournier AE (2008) NeuriteTracer: a novel ImageJ plugin for automated quantification of neurite outgrowth. J Neurosci Methods 168:134-139.

36. Lee IS, Jung K, Kim IS, Lee H, Kim M, Yun S, Hwang K, Shin JE, Park KI (2015) Human neural stem cells alleviate Alzheimer-like pathology in a mouse model. Mol Neurodegener 10:38.

37. Ma W, Bisby MA (2000) Partial sciatic nerve ligation induced more dramatic increase of neuropeptide Y immunoreactive axonal fibers in the gracile nucleus of middle-aged rats than in young adult rats. J Neurosci Res 60:520-530.

38. Peng W, Cotrina ML, Han X, Yu H, Bekar L, Blum L, Takano T, Tian GF, Goldman SA, Nedergaard M (2009) Systemic administration of an antagonist of the ATP-sensitive receptor P2X7 improves recovery after spinal cord injury. Proc Natl Acad Sci U S A 106:12489-12493.

39. Ramadan WS, Abdel-Hamid GA, Al-Karim S, Abbas AT (2017) Histological, immunohistochemical and ultrastructural study of secondary compressed spinal cord injury in a rat model. Folia Histochem Cytobiol 55:11-20.

40. Bareyre FM, Kerschensteiner M, Raineteau O, Mettenleiter TC, Weinmann O, Schwab ME (2004) The injured spinal cord spontaneously forms a new intraspinal circuit in adult rats. Nat Neurosci 7:269-277.

41. Chaplan SR, Bach FW, Pogrel JW, Chung JM, Yaksh TL (1994) Quantitative assessment of tactile allodynia in the rat paw. J Neurosci Methods 53:55-63.

42. Akesson E, Piao JH, Samuelsson EB, Holmberg L, Kjaeldgaard A, Falci S, Sundström E, Seiger A (2007) Long-term culture and neuronal survival after intraspinal transplantation of human spinal cord-derived neurospheres. Physiol Behav 92:6066.

43. Tetzlaff W, Okon EB, Karimi-Abdolrezaee S, Hill CE, Sparling JS, Plemel JR, Plunet WT, Tsai EC, Baptiste D, Smithson LJ, Kawaja MD, Fehlings MG, Kwon BK (2011) A systematic review of cellular transplantation therapies for spinal cord injury. J Neurotrauma 28:1611-1682.

44. Shin JE, Jung K, Kim M, Hwang K, Lee H, Kim IS, Lee BH, Lee IS, Park KI (2018) Brain and spinal cord injury repair by implantation of human neural progenitor cells seeded onto polymer scaffolds. Exp Mol Med 50:39

45. Mukhamedshina YO, Shaymardanova GF, Garanina EE, Salafutdinov II, Rizvanov AA, Islamov RR, Chelyshev YA (2016) Adenoviral vector carrying glial cell-derived neurotrophic factor for direct gene therapy in comparison with human umbilical cord blood cell-mediated therapy of spinal cord injury in rat. Spinal Cord 54:347-359.

46. Sakurai M, Abe K, Hayashi T, Setoguchi Y, Yaginuma G, Meguro T, Tabayashi K (2000) Adenovirus-mediated glial cell line-derived neurotrophic factor gene delivery reduces motor neuron injury after transient spinal cord ischemia in rabbits. J Thorac Cardiovasc Surg 120:1148-1157.

47. Koelsch A, Feng Y, Fink DJ, Mata M (2010) Transgene-mediated GDNF expression enhances synaptic connectivity and GABA transmission to improve functional outcome after 
spinal cord contusion. J Neurochem 113:143-152.

48. Tai MH, Cheng H, Wu JP, Liu YL, Lin PR, Kuo JS, Tseng CJ, Tzeng SF (2003) Gene transfer of glial cell line-derived neurotrophic factor promotes functional recovery following spinal cord contusion. Exp Neurol 183:508-515.

49. Tang XQ, Wang Y, Huang ZH, Han JS, Wan Y (2004) Adenovirus-mediated delivery of GDNF ameliorates corticospinal neuronal atrophy and motor function deficits in rats with spinal cord injury. Neuroreport 15:425-429.

50. Gowing G, Svendsen S, Svendsen CN (2017) Ex vivo gene therapy for the treatment of neurological disorders. Prog Brain Res 230:99-132.

51. Deng LX, Deng P, Ruan Y, Xu ZC, Liu NK, Wen X, Smith GM, Xu XM (2013) A novel growth-promoting pathway formed by GDNF-overexpressing Schwann cells promotes propriospinal axonal regeneration, synapse formation, and partial recovery of function after spinal cord injury. J Neurosci 33:5655-5667.

52. Chen BK, Madigan NN, Hakim IS, Dadsetan M, McMahon SS, Yaszemski MJ, Windebank AJ (2018) GDNF Schwann cells in hydrogel scaffolds promote regional axon regeneration, remyelination and functional improvement after spinal cord transection in rats. J Tissue Eng Regen Med 12:e398e407.

53. Shahrezaie M, Mansour RN, Nazari B, Hassannia H, Hosseini F, Mahboudi H, Eftekhary M, Kehtari M, Veshkini A, Ahmadi Vasmehjani A, Enderami SE (2017) Improved stem cell therapy of spinal cord injury using GDNF-overexpressed bone marrow stem cells in a rat model. Biologicals 50:73-80.

54. Macias MY, Syring MB, Pizzi MA, Crowe MJ, Alexanian AR, Kurpad SN (2006) Pain with no gain: allodynia following neural stem cell transplantation in spinal cord injury. Exp Neurol 201:335-348.

55. Behrstock S, Ebert A, McHugh J, Vosberg S, Moore J, Schneider B, Capowski E, Hei D, Kordower J, Aebischer P, Svendsen CN (2006) Human neural progenitors deliver glial cell linederived neurotrophic factor to parkinsonian rodents and aged primates. Gene Ther 13:379-388.

56. Klein SM, Behrstock S, McHugh J, Hoffmann K, Wallace K, Suzuki M, Aebischer P, Svendsen CN (2005) GDNF delivery using human neural progenitor cells in a rat model of ALS. Hum Gene Ther 16:509-521.

57. Akhtar AA, Gowing G, Kobritz N, Savinoff SE, Garcia L, Saxon D, Cho N, Kim G, Tom CM, Park H, Lawless G, Shelley BC, Mattis VB, Breunig JJ, Svendsen CN (2018) Inducible expression of GDNF in transplanted iPSC-derived neural progenitor cells. Stem Cell Reports 10:1696-1704.

58. Hill CE, Beattie MS, Bresnahan JC (2001) Degeneration and sprouting of identified descending supraspinal axons after contusive spinal cord injury in the rat. Exp Neurol 171:15369.

59. Zhou L, Shine HD (2003) Neurotrophic factors expressed in both cortex and spinal cord induce axonal plasticity after spinal cord injury. J Neurosci Res 74:221-226.

60. Iannotti C, Li H, Yan P, Lu X, Wirthlin L, Xu XM (2003) Glial cell line-derived neurotrophic factor-enriched bridging transplants promote propriospinal axonal regeneration and enhance myelination after spinal cord injury. Exp Neurol 183:379-393.

61. Boucher TJ, Okuse K, Bennett DL, Munson JB, Wood JN, McMahon SB (2000) Potent analgesic effects of GDNF in neuropathic pain states. Science 290:124-127.

62. Mukhida K, Mendez I, McLeod M, Kobayashi N, Haughn C, Milne B, Baghbaderani B, Sen A, Behie LA, Hong M (2007) Spinal GABAergic transplants attenuate mechanical allodynia in a rat model of neuropathic pain. Stem Cells 25:28742885 .

63. Jergova S, Hentall ID, Gajavelli S, Varghese MS, Sagen J (2012) Intraspinal transplantation of GABAergic neural progenitors attenuates neuropathic pain in rats: a pharmacologic and neurophysiological evaluation. Exp Neurol 234:39-49.

64. Kim DS, Jung SJ, Nam TS, Jeon YH, Lee DR, Lee JS, Leem JW, Kim DW (2010) Transplantation of GABAergic neurons from ESCs attenuates tactile hypersensitivity following spinal cord injury. Stem Cells 28:2099-2108.

65. Lee JW, Jergova S, Furmanski O, Gajavelli S, Sagen J (2012) Predifferentiated GABAergic neural precursor transplants for alleviation of dysesthetic central pain following excitotoxic spinal cord injury. Front Physiol 3:167.

66. Alizadeh A, Karimi-Abdolrezaee S (2016) Microenvironmental regulation of oligodendrocyte replacement and remyelination in spinal cord injury. J Physiol 594:3539-3552.

67. Oudega M, Xu XM (2006) Schwann cell transplantation for repair of the adult spinal cord. J Neurotrauma 23:453-467.

68. Li N, Leung GK (2015) Oligodendrocyte precursor cells in spinal cord injury: a review and update. Biomed Res Int 2015:235195.

69. Iwase T, Jung CG, Bae H, Zhang M, Soliven B (2005) Glial cell line-derived neurotrophic factor-induced signaling in Schwann cells. J Neurochem 94:1488-1499. 